\title{
Strong-coupling phases of frustrated bosons on a two-leg ladder with ring exchange
}

\author{
D. N. Sheng, ${ }^{1}$ Olexei I. Motrunich, ${ }^{2}$ Simon Trebst, ${ }^{3}$ Emanuel Gull, ${ }^{4}$ and Matthew P. A. Fisher ${ }^{3}$ \\ ${ }^{1}$ Department of Physics and Astronomy, California State University, Northridge, California 91330, USA \\ ${ }^{2}$ Department of Physics, California Institute of Technology, Pasadena, California 91125, USA \\ ${ }^{3}$ Microsoft Research, Station Q, University of California, Santa Barbara, California 93106, USA \\ ${ }^{4}$ Theoretische Physik, Eidgenössische Technische Hochschule Zürich, CH-8093 Zürich, Switzerland
}

(Received 7 May 2008; published 26 August 2008)

\begin{abstract}
Developing a theoretical framework to access the quantum phases of itinerant bosons or fermions in two dimensions that exhibit singular structure along surfaces in momentum space but have no quasiparticle description remains a central challenge in the field of strongly correlated physics. In this paper we propose that distinctive signatures of such two-dimensional (2D) strongly correlated phases will be manifest in quasi-onedimensional " $N$-leg ladder" systems. Characteristic of each parent 2D quantum liquid would be a precise pattern of one-dimensional (1D) gapless modes on the $N$-leg ladder. These signatures could be potentially exploited to approach the 2D phases from controlled numerical and analytical studies in quasi-one-dimension. As a first step we explore itinerant-boson models with a frustrating ring-exchange interaction on the two-leg ladder, searching for signatures of the recently proposed two-dimensional $d$-wave-correlated Bose liquid (DBL) phase. A combination of exact diagonalization, density-matrix renormalization-group, variational Monte Carlo, and bosonization analysis of a quasi-1D gauge theory provide compelling evidence for the existence of an unusual strong-coupling phase of bosons on the two-leg ladder, which can be understood as a descendant of the two-dimensional DBL. We suggest several generalizations to quantum spin and electron Hamiltonians on ladders, which could likewise reveal fingerprints of such 2D non-Fermi-liquid phases.
\end{abstract}

DOI: $10.1103 /$ PhysRevB.78.054520

PACS number(s): 71.10.Hf, 71.10.Pm, 74.20.Mn, 75.10.Jm

\section{INTRODUCTION}

During the past decade it has become abundantly clear that there are several subclasses of two-dimensional (2D) spin liquids. ${ }^{1}$ Perhaps best understood are the topological spin liquids that support gapped excitations which carry fractional quantum numbers. ${ }^{2-5}$ Another possibility are gapless spin liquids with no topological structure. These gapless spin liquids will generically exhibit spin correlations that decay as a power law in space and which can oscillate at particular wave vectors. In such "algebraic" or "critical" spin liquids, ${ }^{1,6-8}$ these wave vectors are limited to a finite discrete set. Their effective field theories will often exhibit a relativistic structure-as is the case when the spinons in a slaveparticle construction are massless Dirac fermions coupled to a U(1) gauge field. However, it is possible that the spin correlations exhibit singularities along surfaces in momentum space. These are analogous to the Fermi surfaces in a Fermi liquid. However in such "quantum spin metals" a quasiparticle picture will presumably be inapplicable. Spin liquids with a spinon Fermi surface are examples of particular interest and have been studied in a number of works ${ }^{1,9-13}$ - most recently as a candidate for a spin-liquid phase $\mathrm{e}^{14-16}$ in the organic compound $\kappa-(\mathrm{ET})_{2} \mathrm{Cu}_{2}(\mathrm{CN})_{3}$.

Developing a theoretical framework for itinerant 2D nonFermi liquids is arguably even more challenging than for spin liquids. Towards this end a recent work ${ }^{17}$ explored the possibility of an uncondensed quantum phase of itinerant bosons in two dimensions which is a conducting fluid but not superfluid. Because of the characteristic nodal structure observed by moving one boson around another, Ref. 17 called such phases " $d$-wave-correlated Bose liquids" (DBLs). Various physical correlators were found to be singular across surfaces in momentum space in the DBL. For example, the boson occupation number $n(\mathbf{q})$ is singular at some "Bose surfaces" such as those illustrated in Fig. 1. Other examples with critical surfaces were studied recently in Ref. 18 .

The central idea underlying this paper is that $2 \mathrm{D}$ phases of quantum spins and itinerant fermions or bosons with singular surfaces in momentum space should have definite signatures when restricted to a quasi-one-dimensional (1D) geometry, e.g., when the system is placed onto an $N$-leg ladder. To be concrete, suppose we have a singular 2D surface in momentum space in the ground state of some square lattice quantum Hamiltonian. If we put the Hamiltonian on an $\mathrm{N}$-leg ladder,

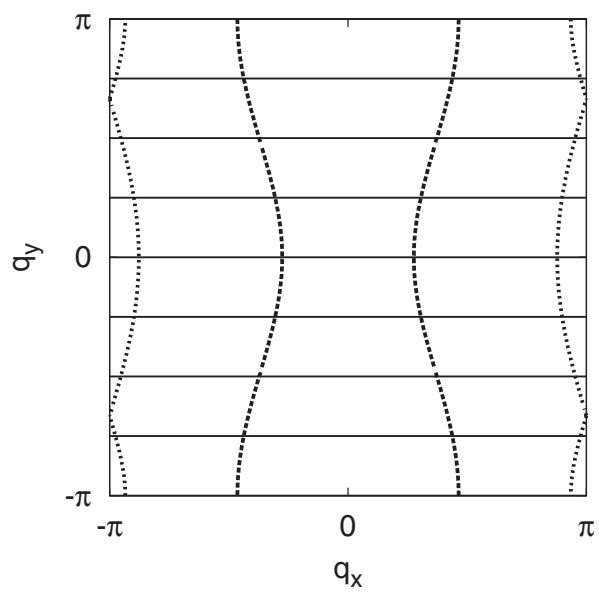

FIG. 1. Schematics of discrete $q_{y}=2 \pi / N$ cuts through Bose surfaces in some anisotropic 2D system placed on an $N$-leg ladder. Here for illustration $N=8$. Such Bose surfaces appear in a DBL construction described in the text. To avoid clutter, we do not show the Fermi surfaces of the $d_{1}$ and $d_{2}$ slave particles. 
the transverse momentum $q_{y}$ becomes discrete. With large $N$ one would expect that the short-range energetics that stabilizes the $2 \mathrm{D}$ phase will still be present on the ladder and will lead to a set of $1 \mathrm{D}$ gapless modes, where the discrete momenta cut through the singular surface, as illustrated in Fig. 1.

The long-wavelength physics of these 1D modes will be described in terms of some multichannel Luttinger liquid, whose behavior is different from that of the parent 2D state. However, the number of gapless 1D modes and their respective momenta characterizing the oscillatory power-law decays would provide a distinctive "fingerprint" of the parent 2D liquid. For example, the number of gapless 1D modes would grow linearly with $N$ and the sum of their momenta could satisfy some generalized Luttinger volume. Moreover, these signatures could be potentially exploited to approach the 2D phases from controlled numerical and analytical studies in quasi-one-dimension. Indeed, as we demonstrate explicitly in this paper, the (putative) singular surface of the 2D DBL phase is already manifest on the two-leg ladder.

The itinerant uncondensed DBL of Ref. 17 is constructed by writing a boson in terms of fermionic partons,

$$
b^{\dagger}=d_{1}^{\dagger} d_{2}^{\dagger},
$$

and considering uncoupled mean-field states of $d_{1}$ and $d_{2}$. Here and below, we study hard-core bosons. The DBL phase on a square lattice is obtained by taking the $d_{1}$ fermion to hop preferentially along the $\hat{x}$ axis and the $d_{2}$ fermions to hop preferentially along the $\hat{y}$ axis. One can enforce the square lattice symmetry in the boson state by requiring that the $d_{1}$ and $d_{2}$ hopping patterns are related by a $90^{\circ}$ rotation. To recover the physical Hilbert space requires the condition $d_{1}^{\dagger} d_{1}=d_{2}^{\dagger} d_{2}=b^{\dagger} b$ at each site. Within a Gutzwiller projection approach, the corresponding boson wave function is taken as product of two fermion Slater determinants,

$$
\Psi_{\text {bos }}\left(r_{1}, r_{2}, \ldots\right)=\Psi_{d_{1}}\left(r_{1}, r_{2}, \ldots\right) \cdot \Psi_{d_{2}}\left(r_{1}, r_{2}, \ldots\right) .
$$

In the DBL the $d_{1}$ and $d_{2}$ Fermi surfaces are different, compressed in the $\hat{x}$ and $\hat{y}$ directions, respectively. This results in a characteristic $d$-wave-like nodal structure observed when moving one boson around another in $\Psi_{\text {bos. }}$. The boson momentum distribution function $n(\mathbf{q})$ in the DBL is singular on two surfaces that are constructed from the $d_{1}$ and $d_{2}$ Fermi surfaces as enveloping $\boldsymbol{k}_{F 1} \pm \boldsymbol{k}_{F 2}$ surfaces. More details are found in Ref. 17. Figure 1 shows one example of the $\boldsymbol{k}_{F 1} \pm \boldsymbol{k}_{F 2}$ loci for an open $d_{1}$ Fermi surface and a closed $d_{2}$ Fermi surface. (The Fermi surfaces themselves are not shown; this DBL state would be anisotropic on the square lattice but is closer to the ladder states considered here where there is no $90^{\circ}$ rotation symmetry.) As an alternative to the Gutzwiller construction, one can project into the physical Hilbert space within a gauge theory approach, coupling $d_{1}$ and $d_{2}$ with opposite gauge charges to an emergent $\mathrm{U}(1)$ gauge field.

Reference 17 also proposed a frustrated hard-core boson model with ring exchanges that can potentially realize such DBL phases:

$$
H=H_{J}+H_{\text {ring }},
$$

$$
\begin{gathered}
H_{J}=-J \sum_{\mathbf{r} ; \hat{\mu}=\hat{x}, \hat{y}}\left(b_{\mathbf{r}}^{\dagger} b_{\mathbf{r}+\hat{\mu}}+\text { H.c. }\right), \\
H_{\text {ring }}=K \sum_{\mathbf{r}}\left(b_{\mathbf{r}}^{\dagger} b_{\mathbf{r}+\hat{x}} b_{\mathbf{r}+\hat{x}+\hat{y}}^{\dagger} b_{\mathbf{r}+\hat{y}}+\text { H.c. }\right),
\end{gathered}
$$

with $J, K>0$. In addition to the usual boson hopping term, this Hamiltonian contains a four-site ring term acting on each square plaquette. With $K$ positive this term is "frustrating," violating the Marshall sign rule of the hopping termmaking the system intractable by quantum Monte Carlo simulations. This boson Hamiltonian was constructed ${ }^{17}$ by taking the strong-coupling limit of the lattice gauge theory for the $d_{1}$ and $d_{2}$ fermions. Increasing the disparity in the fermion hopping along the $\hat{x}$ and $\hat{y}$ axes corresponds to an increase in $K$.

In this paper, we make a first step in the proposed program of ladder studies by exploring the ring model [Eq. (3)] on a two-leg ladder. Of course, the picture of discrete $q_{y}$ cuts through two-dimensional surfaces is pushed to the extreme here. Nevertheless and quite remarkably, in a wide parameter regime of intermediate to large ring couplings, we find an unusual phase which can be understood via the DBL construction [Eq. (2)]. This is a strong-coupling phase with no perturbative picture in terms of the original bosons. The slave-particle approach provides a new starting point, and the resulting gauge theory of the DBL can be solved by the conventional $1 \mathrm{D}$ bosonization tools, ${ }^{19-22}$ providing a consistent picture of the unusual phase.

The paper is organized as follows: Section II sets the stage by describing DBL states on the two-leg ladder. Section III presents the results of exact numerical studies of the $J-K$ model. The focus here is on the DBL phase that emerges when the conventional " $q=0$ liquid" is destroyed by the ring exchanges. However, the phase diagram is even richer and contains two more unusual phases in which bosons are paired. One of these phases can be accessed analytically as an instability of yet a different DBL phase. Appendix A summarizes the technical gauge theory description of the DBL phases, while Appendix B presents simple analytical results for the ring model in some limiting cases. Appendix $\mathrm{C}$ constructs trial wave functions for the boson-paired phases. Section IV concludes with an outline of future program of ladder studies.

\section{DBL STATES FOR HARD-CORE BOSONS ON A TWO-LEG LADDER}

The DBL construction [Eq. (2)] proceeds by taking distinct hopping ground states for the $d_{1}$ and $d_{2}$ fermions. The hopping patterns of the two fermion species can be independent and need to only respect the ladder symmetries. There is a bonding and an antibonding band for each species. We take the $d_{1}$ fermions to hop more strongly along the chains and always assume that the chemical potential crosses both bands as illustrated in Fig. 2. We take the $d_{2}$ fermions to hop more strongly on the rungs and consider two cases shown in the bottom panels of Fig. 2: the "DBL[2,1] phase," where only the bonding band of the $d_{2}$ fermions crosses the Fermi level, 


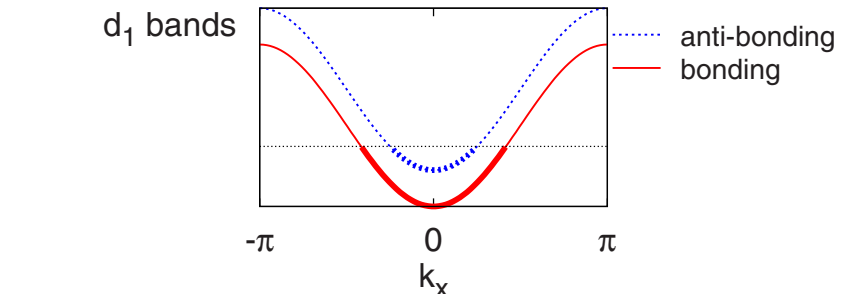

$d_{2}$ bands for $D B L[2,1]$
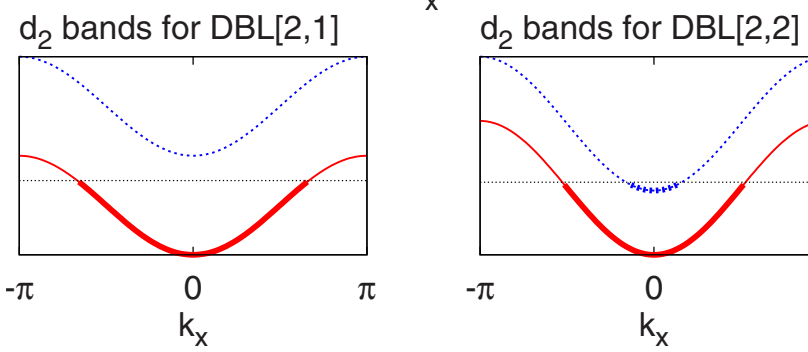

FIG. 2. (Color online) $d_{1}$ and $d_{2}$ bands for the DBL construction on the two-leg ladder. Top panel: $d_{1}$ fermions partially occupy both the bonding and the antibonding bands. Bottom left: In the DBL[2,1] phase, $d_{2}$ fermions occupy only the bonding band. Bottom right: In the DBL[2,2] phase, $d_{2}$ fermions partially occupy both bands. This state is likely unstable toward a boson-paired phase, as discussed in Appendix A 3. In the wave-function construction, only the occupied orbitals and not the band dispersions are important.

and the "DBL[2,2] phase," where both $d_{2}$ bands are partially occupied. The first and second arguments in the square brackets refer to the numbers of involved $d_{1}$ and $d_{2}$ bands, respectively.

Let us first discuss the properties of the DBL[2,1] phase. We denote the Fermi momenta of the $d_{1}$ bonding and antibonding bands as $k_{F 1}^{(0)}$ and $k_{F 1}^{(\pi)}$, corresponding to the transverse momenta $k_{y}=0$ and $k_{y}=\pi$, respectively (which is a convenient alternative to the quantum number for the leg interchange symmetry). We denote the Fermi momentum of the $d_{2}$ bonding band as $k_{F 2}$ (there is no $d_{2}$ antibonding occupation in the DBL[2,1]). The Fermi momenta satisfy $k_{F 1}^{(0)}$ $+k_{F 1}^{(\pi)}=k_{F 2}=2 \pi \rho$, where $\rho$ is the boson density per site and remembering that there are two sites in each rung, while the lattice spacing is set to 1 .

In a mean-field treatment, the boson Green's function is a product of two fermion Green's functions and therefore oscillates at different possible $k_{F 1} \pm k_{F 2}$ wave vectors and decays as $1 / x^{2}$. Going beyond the mean field as described in Appendix A, we expect that the oscillations at wave vectors $q_{x}^{(0 / \pi)}=k_{F 2}-k_{F 1}^{(0 / \pi)}$ have slower power-law decay, while the other two wave vectors $k_{F 2}+k_{F 1}^{(0 / \pi)}$ have faster power-law decay than the mean field. The complete result is, dropping all amplitudes,

$$
\begin{gathered}
G_{b}(\mathbf{r}) \sim \frac{\cos \left[\left(k_{F 2}-k_{F 1}^{(0)}\right) x\right]}{x^{2-\eta^{(0)}}}+e^{i \pi y} \frac{\cos \left[\left(k_{F 2}-k_{F 1}^{(\pi)}\right) x\right]}{x^{2-\eta^{(\pi)}}} \\
+\frac{\cos \left[\left(k_{F 2}+k_{F 1}^{(0)}\right) x\right]}{x^{2+\cdots}}+e^{i \pi y} \frac{\cos \left[\left(k_{F 2}+k_{F 1}^{(\pi)}\right) x\right]}{x^{2+\cdots}} .
\end{gathered}
$$

The first line shows the enhanced correlators. These can be guessed using an "Amperean rule" mnemonic inherited from the fermion-gauge studies in two dimensions and verified by the bosonization solution in Appendix A: Composites that involve fermion bilinears with opposite group velocities but produce parallel gauge currents are enhanced by the gauge fluctuations. Thus, low-energy "bosons" $d_{1 L}^{(0 / \pi) \dagger} d_{2 R}^{\dagger}$ carrying $q_{x}^{(0 / \pi)}$ are expected to be enhanced. The contributions in the second line, on the other hand, can be shown to be always suppressed beyond the mean field. Appendix A presents a complete low-energy theory of the DBL[2,1] which-once the gauge fluctuations are treated-contains two phonon modes and in principle allows calculation of all exponents. Since the power laws are affected also by short-range interactions and there are many parameters allowed in the theory, we do not pursue this calculation in general but mainly rely on the Amperean rules that capture the gauge fluctuation effects. This is reasonable if the gauge interactions dominate.

The boson singularities at nonzero wave vectors become manifest upon Fourier transformation of $G_{b}(\mathbf{r})$ to obtain the boson occupation number $n(\mathbf{q})$. For example, at the enhanced wave vectors $\left(q_{x}^{(0)}, 0\right)$ and $\left(q_{x}^{(\pi)}, \pi\right)$, we have

$$
n\left(q_{x}^{\left(k_{y}\right)}+\delta q_{x}, k_{y}\right) \sim\left|\delta q_{x}\right|^{1-\eta^{\left(k_{y}\right)} .}
$$

The boson density correlations are analyzed similarly. In a naive mean field, $D_{b}(\mathbf{r})=\langle n(\mathbf{r}) n(0)\rangle \sim D_{d 1}(\mathbf{r})+D_{d 2}(\mathbf{r})$, which oscillates at various " $2 k_{F}$ " vectors and decays as $1 / x^{2}$. Beyond the mean field, the power laws are modified:

$$
\begin{gathered}
D_{d 1}(\mathbf{r}) \sim \frac{1}{x^{2}}+\frac{\cos \left[2 k_{F 1}^{(0)} x\right]}{x^{2-\cdots}}+\frac{\cos \left[2 k_{F 1}^{(\pi)} x\right]}{x^{2-\cdots}} \\
+e^{i \pi y} \frac{\cos \left[\left(k_{F 1}^{(0)}+k_{F 1}^{(\pi)}\right) x\right]}{x^{2-\cdots}}+e^{i \pi y} \frac{\cos \left[\left(k_{F 1}^{(0)}-k_{F 1}^{(\pi)}\right) x\right]}{x^{2+\cdots}}, \\
D_{d 2}(\mathbf{r}) \sim \frac{1}{x^{2}}+\frac{\cos \left[2 k_{F 2} x\right]}{x^{2-\cdots}} .
\end{gathered}
$$

The distinct momenta are $\left(2 k_{F 1}^{(0)}, 0\right),\left(2 k_{F 1}^{(\pi)}, 0\right),\left(2 k_{F 2}, 0\right)$, $\left(k_{F 1}^{(0)}+k_{F 1}^{(\pi)}, \pi\right),\left(k_{F 1}^{(0)}-k_{F 1}^{(\pi)}, \pi\right)$, and $(0,0)$. The first four involve a particle and a hole of the same species but with opposite group velocities and are therefore expected to be enhanced by the gauge fluctuations. In the above equations, this is indicated schematically with $1 / x^{2-\cdots}$. However, these correlators can also be affected-either enhanced or suppressed-by short-range interactions. Without knowing all parameters in the theory, we cannot calculate the exponents reliably. The oscillation at the wave vector $\left(k_{F 1}^{(0)}\right.$ $\left.-k_{F 1}^{(\pi)}, \pi\right)$ can be shown to be always suppressed beyond the mean field. Finally, the zero-momentum power law remains unchanged.

More details on the DBL[2,1] phase can be found in Appendix A. In particular, we show that the two-boson Green's function exhibits some internal $d$-wave character, which originates from the nontrivial wave-function signs. It is these signs that prompted the name " $d$-wave Bose liquid" in the 2D continuum setting, ${ }^{17}$ where the wave function goes through a sequence of signs +-+- upon taking one particle around another. However, such correlations do not necessarily mean that the system is near a $d$-wave-paired phase. For 
example, we also examine a potential instability of the $\operatorname{DBL}[2,1]$ driven by an allowed nonmarginal four-fermion term. We find that the resulting phase is a boson-paired liquid with an internal $s$-wave character.

Let us briefly mention the DBL[2,2] case, where in the mean field both $d_{1}$ and $d_{2}$ have partial occupations of both the bonding and the antibonding bands. One can calculate various correlations in the mean field as done before, and the structure is richer since there is an additional band present. Some details are given in Appendix A 3. Beyond the mean field but ignoring nonmarginal four-fermion terms, this phase would have three gapless modes. However, an analysis of allowed interactions suggests that this phase has a strong instability toward a boson-paired phase with an $s$-wave character. Remarkably, as we will see in Sec. III, a boson-paired phase with similar properties is found in the ring model in the regime of small interchain coupling $J_{\perp}$, where we initially hoped to find the DBL[2,2] phase. Thus, the slaveparticle formulation and the gauge theory description solved via subsequent bosonization open a nonperturbative access to this interesting boson-paired phase.

\section{FRUSTRATED $J$-K MODEL ON THE TWO-LEG LADDER}

We study the ring model [Eq. (3)] on a two-leg ladder with a boson hopping term $J$ that allows different amplitudes along and perpendicular to the chains:

$$
H_{J}=-J \sum_{\mathbf{r}}\left(b_{\mathbf{r}}^{\dagger} b_{\mathbf{r}+\hat{x}}+\text { H . c. }\right)-J_{\perp} \sum_{x}\left(b_{(x, 1)}^{\dagger} b_{(x, 2)}+\text { H . c. }\right),
$$

where $\mathbf{r}=(x, y)$ represent integer lattice sites. We take $y$ $=1, \ldots, L_{y}$, with $L_{y}=2$ and $x=1, \ldots, L_{x}$, with $L_{x}$ as the length of the two-leg system. The ring terms are associated with the square plaquettes of the two-leg ladder. Our convention here and in all numerical work below is that each rung hopping term and each plaquette ring-exchange term is counted precisely once.

We use exact diagonalization (ED) and density-matrix renormalization-group (DMRG) (Refs. 23 and 24) methods supplemented with trial wave-function variational Monte Carlo (VMC) (Ref. 25) to determine the nature of the ground state of Hamiltonian (3) at boson filling number $\rho=\frac{N_{b}}{L_{x} L_{y}}$, where $N_{b}$ is the total number of bosons in the system. The obtained phase diagrams at densities $\rho=1 / 3$ and $1 / 9$ are shown in Figs. 3 and 4, respectively. These are typical results for the boson ring model away from half filling.

Using ED and DMRG, we find four different quantum phases upon increasing the ring-exchange coupling $K$ and varying the interchain coupling $J_{\perp}$. At small $K$, the "superfluid" phase (SF) with $\mathbf{q}=\mathbf{0}$ quasi-long-range order (QLRO) is stable for both filling numbers. The DBL phase, which is produced by the ring exchanges, dominates the intermediate parameter space at higher filling number $\rho=1 / 3$, while it occupies a substantially smaller region at $\rho=1 / 9$. Interestingly, at $\rho=1 / 3$ and small $J_{\perp}$, an $s$-wave-paired phase emerges between the SF and DBL as a consequence of the

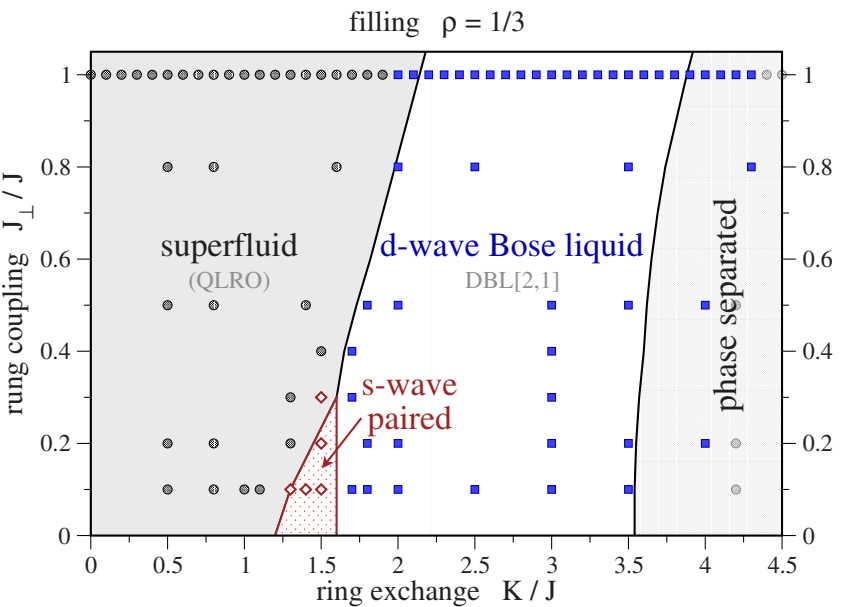

FIG. 3. (Color online) Phase diagram for the two-leg boson system at filling $\rho=1 / 3$. The symbols represent DMRG estimates with shaded gray circles indicating the superfluid phase, the filled blue squares the DBL, and the open red diamonds the $s$-wave-paired state. The schematic line boundaries are obtained from VMC calculations.

competition between the boson hopping and ring-exchange terms. At $\rho=1 / 9$ and small $J_{\perp}$, a $d$-wave-paired phase is found adjacent to the DBL. Finally, for strong ring exchanges, phase separation eventually wins near $K \sim 4 J$ for both fillings, separating into a region with $\rho=1 / 2$ and an empty region $\rho=0$. The characteristic features of each phase will be discussed in Secs. III A-III E.

Our complementary VMC study is centered around the DBL phase. The DBL wave function [Eq. (2)] is constructed from the slave-particle formulation and thus allows us to compare the exact ED/DMRG results and the gauge theory description. Jastrow SF and boson-paired trial wave functions (described in Appendix C) are also considered in order to better understand the exact numerical results. We note that

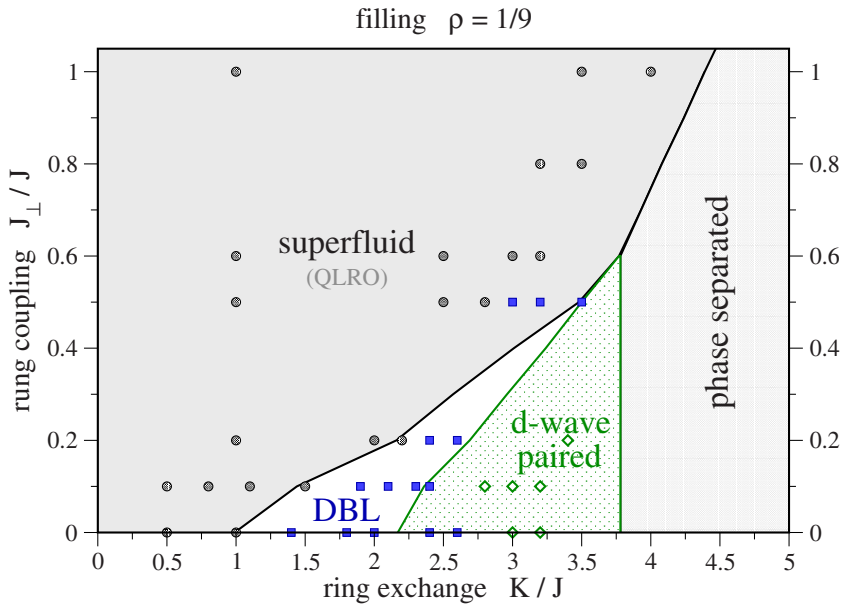

FIG. 4. (Color online) Phase diagram for the two-leg boson system at filling $\rho=1 / 9$. The symbols indicate DMRG estimates for the various phases with shaded gray circles for the superfluid phase, the filled blue squares for the DBL, and the open green diamonds for the $d$-wave-paired state. The schematic line boundaries are obtained from VMC calculations. 


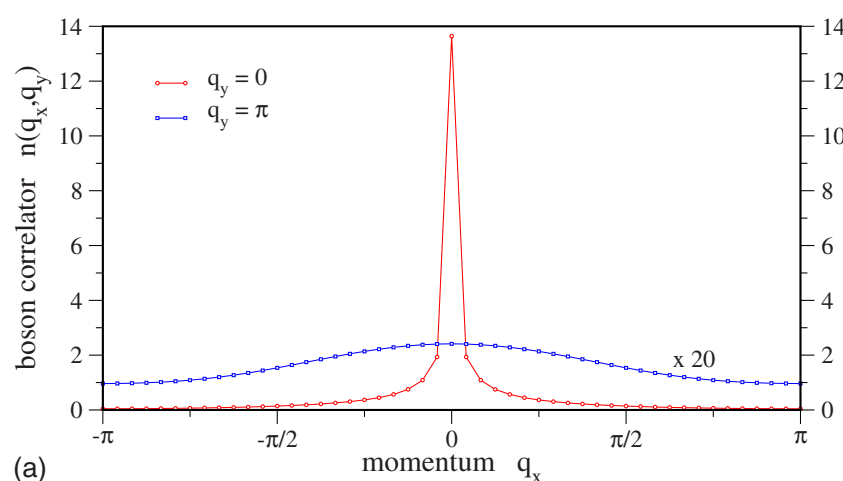

(a)

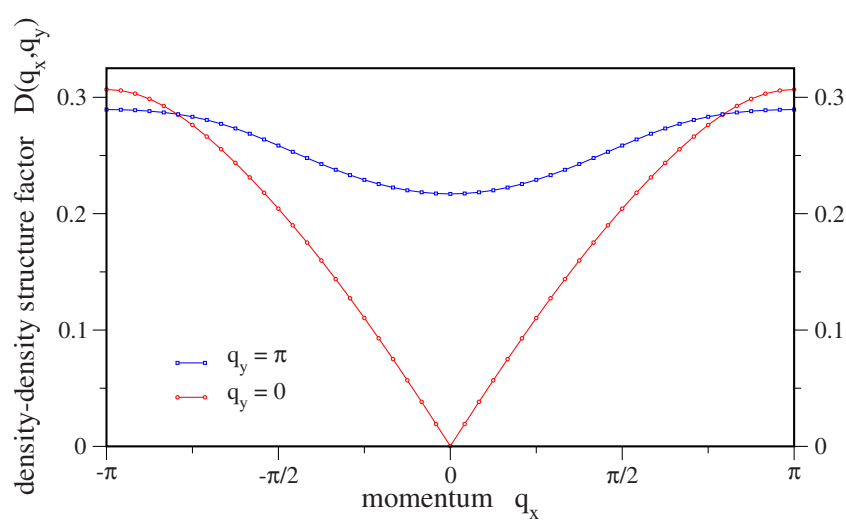

(b)

FIG. 5. (Color online) DMRG results for (a) the boson occupation number $n(\mathbf{q})$ and (b) the density-density structure factor $D(\mathbf{q})$ for the two-leg system at $\rho=1 / 3, J_{\perp}=J, K=J$; the system length is $L_{x}=48$. In (a), the $q_{y}=\pi$ data were multiplied by a factor of 20 . The results are representative for the superfluid phase.

this variational approach has been rather successful in one dimension, e.g., in the original studies of the 1D $t$ - $J$ model. ${ }^{26-28}$ We borrow some ideas from these studies such as allowing the fermionic determinants to also play a Jastrow role via det $\rightarrow \operatorname{sgn}($ det $)|\operatorname{det}|^{p}$, where $p$ is a variational parameter. In our ring model, the phase boundary estimated from VMC almost coincides with the one determined from DMRG as indicated in Fig. 3 for $\rho=1 / 3$ filling. At smaller filling number, DMRG and VMC also find the same topology of the phase diagram as given in Fig. 4.

We first describe measurements in the ground state. Imposing periodic boundary conditions in the $\hat{x}$ direction in our numerical calculations, we can characterize the ED states by a momentum $k_{x}$. In our DMRG calculations we work with real-valued wave functions which give no ambiguity when the system exhibits a unique ground state that caries zero momentum. If the ground state carries nontrivial momentum $k_{x}$, its time-reversed partner carries $-k_{x}$, and the DMRG state is some real-valued combination of these. While the latticespace measurements may depend slightly on the specific combination (but vanishingly in the large system limit), the momentum space measurements $n(\mathbf{q})$ and $D(\mathbf{q})$ described below are unique and insensitive to this. In our DMRG calculations, more than 1500 states were kept in each block ${ }^{23,24}$ to ensure accurate results, and the density-matrix truncation error is of the order of $10^{-7}$. The typical error for the ground-

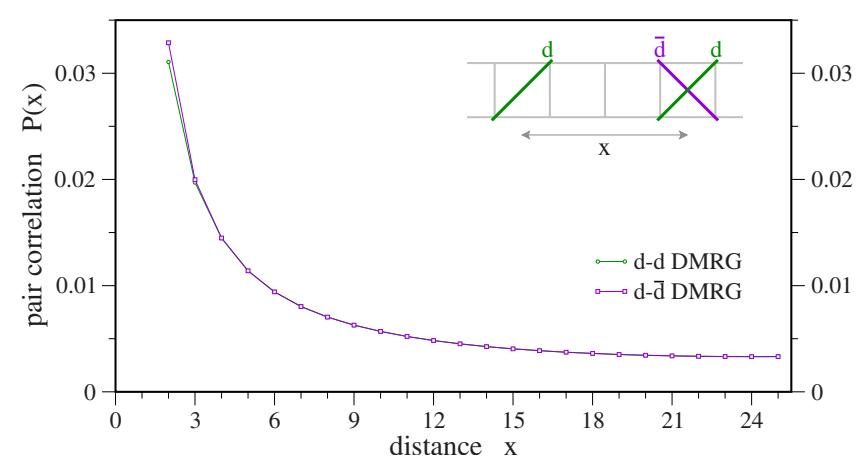

FIG. 6. (Color online) DMRG results for the boson pairing correlations in real space for the SF system described in Fig. 5. We show correlations for pairs on the diagonals $d$ and $\bar{d}$, but roughly similar values are obtained for all pair orientations.

state energy is of the order of $10^{-5}$ for all systems we have studied. The relative error in the correlation functions varies between $10^{-4}$ and $10^{-2}$, depending on the type of the correlations and the spatial distance between operators.

We measure the boson correlator in the ground state,

$$
G_{b}\left(\mathbf{r}, \mathbf{r}^{\prime}\right)=\left\langle b_{\mathbf{r}}^{\dagger} b_{\mathbf{r}^{\prime}}\right\rangle
$$

and its Fourier transform

$$
n(\mathbf{q})=\frac{1}{L_{x} L_{y}} \sum_{\mathbf{r}, \mathbf{r}^{\prime}} G_{b}\left(\mathbf{r}, \mathbf{r}^{\prime}\right) e^{i \mathbf{q} \cdot\left(\mathbf{r}-\mathbf{r}^{\prime}\right)}=\left\langle b_{\mathbf{q}}^{\dagger} b_{\mathbf{q}}\right\rangle,
$$

which is interpreted as mode $\mathbf{q}$ occupation number.

We also measure the boson density correlator

$$
D_{b}\left(\mathbf{r}, \mathbf{r}^{\prime}\right)=\left\langle\left[\rho_{b}(\mathbf{r})-\bar{\rho}\right]\left[\rho_{b}\left(\mathbf{r}^{\prime}\right)-\bar{\rho}\right]\right\rangle,
$$

where $\bar{\rho}$ is the average boson density. The density structure factor is

$$
D_{b}(\mathbf{q})=\frac{1}{L_{x} L_{y}} \sum_{\mathbf{r}, \mathbf{r}^{\prime}} D_{b}\left(\mathbf{r}, \mathbf{r}^{\prime}\right) e^{i \mathbf{q} \cdot\left(\mathbf{r}-\mathbf{r}^{\prime}\right)}=\left\langle\delta \rho_{-\mathbf{q}} \delta \rho_{\mathbf{q}}\right\rangle .
$$

For both the boson or the boson density correlations, a power-law $1 / x^{p}$ envelope in real space gives rise to a singularity $|\delta q|^{p-1}$ in momentum space.

Finally, we measure the two-boson (pairing) correlator,

$$
P_{2 b}\left(\mathbf{r}_{1}, \mathbf{r}_{2} ; \mathbf{r}_{1}^{\prime}, \mathbf{r}_{2}^{\prime}\right)=\left\langle b^{\dagger}\left(\mathbf{r}_{1}\right) b^{\dagger}\left(\mathbf{r}_{2}\right) b\left(\mathbf{r}_{1}^{\prime}\right) b\left(\mathbf{r}_{2}^{\prime}\right)\right\rangle,
$$

which creates a pair of bosons at $\mathbf{r}_{1}, \mathbf{r}_{2}$ and removes a pair at $\mathbf{r}_{1}^{\prime}, \mathbf{r}_{2}^{\prime}$. This is useful for detecting boson-paired phases where the single-boson correlator decays exponentially. In the pairing correlator figures below, we present $P_{2 b}$ for a fixed created $+45^{\circ}$ diagonal pair $\left\{\mathbf{r}_{1}=(0,1), \mathbf{r}_{2}=(1,2)\right\}$ near the origin, while the removed pair is either a $+45^{\circ}$ diagonal $\left\{\mathbf{r}_{1}^{\prime}\right.$ $\left.=(x, 1), \mathbf{r}_{2}^{\prime}=(x+1,2)\right\}$ or a $-45^{\circ}$ diagonal pair $\left\{\mathbf{r}_{1}^{\prime}=(x, 2), \mathbf{r}_{2}^{\prime}\right.$ $=(x+1,1)\}$. We have measured the correlator for other pair orientations as well. However, we find that the diagonaldiagonal correlations are the most distinguishing ones in the two paired phases. When the amplitudes for the $\pm 45^{\circ}$ diagonals have the same sign, we refer to this as $s$-wave character. On the other hand, when the signs are opposite, we call this 

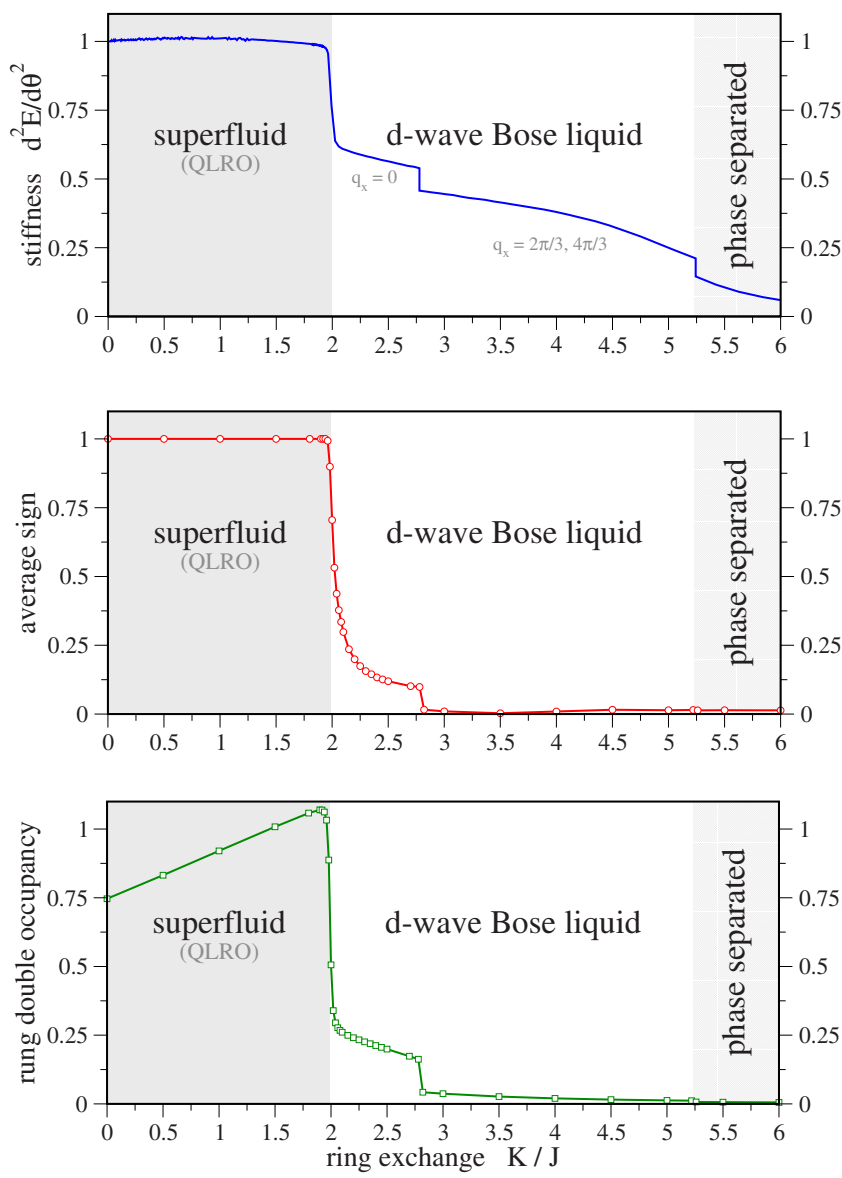

FIG. 7. (Color online) Exact diagonalization results for a system of size $L_{x}=12$ at filling $\rho=1 / 3$ and $J_{\perp}=J$. Shown are the superfluid stiffness (top panel), the average sign of the ground-state wave function (middle panel), and the average double occupancy of rungs (lower panel) as a function of the ring exchange $K$.

$d$-wave character. An extended discussion can be found in Appendix A following Eq. (A13).

\section{A. Superfluid phase}

Let us first discuss the phase at very small $K$. The system is not frustrated in the $K=0$ limit and the ground-state wavefunction (WF) components in the boson occupation number basis should all be of the same sign (positive) to gain the lowest hopping energy. Interestingly, the ED calculations for small $L_{x}=12$ and $L_{x}=18$ systems find that this feature of the WF remains robust up to a finite value of $K \sim 2 J$, close to the stability boundary of the SF phase. This is illustrated in the middle panel of Fig. 7, where the average sign of coefficients in the ground-state wave function is plotted. The boson occupation number $n\left(q_{x}, q_{y}\right)$ has a sharp peak at the wave vector $\mathbf{q}=(0,0)$. This is shown in Fig. 5(a) for a system at $\rho$ $=1 / 3, J_{\perp}=J, K=J$, and length $L_{x}=48$, calculated using the DMRG method. For this system size, the peak value at $\mathbf{q}$ $=(0,0)$ is about 100 times larger than the value at wave vector $\mathbf{q}=(0, \pi)$. This is consistent with the expected singular behavior $n\left(q_{x}, 0\right) \sim\left|q_{x}\right|^{p_{b}-1}$ and nonsingular $n\left(q_{x}, \pi\right)$ in the SF phase with 1D QLRO. The boson correlator decays as
$1 / x^{p_{b}}$ in real space and we estimate $p_{b} \approx 0.25$. As shown in Fig. 5(b) for the same system, the boson density-density structure factor $D\left(q_{x}, 0\right)$ has a $\left|q_{x}\right|$ dependence around $q_{x}=0$, reflecting the global boson number conservation, while $D\left(q_{x}, \pi\right)$ is nonzero and has no visible singularities.

The pairing correlations in real space show a power-law behavior, with nearly equal magnitudes for all pair orientations. This is shown in Fig. 6 , where we create a $+45^{\circ}$ diagonal pair near the origin and remove either a $+45^{\circ}$ diagonal pair or a $-45^{\circ}$ diagonal pair near $x$. Furthermore, the exponent $p_{2 b}$ of the power law $1 / x^{p_{2 b}}$ in the pairing correlator is found to be $p_{2 b} \approx 1$, which is about four times that for the single-boson correlator, $p_{2 b} \approx 4 p_{b}$. Such behavior of the pairing correlations is expected in the SF phase, which has QLRO in the single-boson correlations.

These features characterize the SF phase. It also has a finite $\rho_{s} L$, where $\rho_{s}$ is the "superfluid stiffness," which we can measure in our numerical calculations by imposing a small twist in the boundary conditions. Results from ED of a small system with $L_{x}=12$ are given in the top panel of Fig. 7 and related DMRG measurements for larger systems are discussed in detail in Sec. III B 1.

\section{B. $d$-wave-correlated Bose liquid}

As we further increase the strength of the ring exchange $K$, the system undergoes a (first-order) phase transition where the stiffness $\rho_{s}$ drops steeply by a factor of 2; see Fig. 7 and Sec. III B 1. There is also an abrupt change in the character of the wave-function sign, which we can monitor in the ED analysis for small system sizes by simple counting and shown in the middle panel of Fig. $7 .^{29}$ This phase has unusual phenomenology and can be identified as the DBL[2,1] phase: There is no boson peak at $\mathbf{q}=(0,0)$, but instead the boson occupation number $n\left(q_{x}, q_{y}\right)$ shows singular points at nonzero wave vectors $\left( \pm q_{x}^{(0)}, 0\right)$ and $\left( \pm q_{x}^{(\pi)}, \pi\right)$, as if there is a "condensate" of bonding and antibonding boson modes at these $q_{x}$. Figure 8 presents the characteristic plots of $n\left(q_{x}, q_{y}\right)$ and the density-density structure factor $D\left(q_{x}, q_{y}\right)$ deep in this phase for filling $\rho=1 / 3$ taken at $J_{\perp}$ $=J, K=3 J$, and a system length $L_{x}=48$.

The singular boson wave vectors $q_{x}^{(0)}$ and $q_{x}^{(\pi)}$ in general vary with the parameters $K$ and $J_{\perp}$. An example is shown in Fig. 9 for the system at $\rho=1 / 3$ with $J_{\perp}=J$ and varying $K$. Just into the DBL phase near $K \gtrsim 2$, we find a small $q_{x}^{(0)}$ and a large $q_{x}^{(\pi)}$. In the wave-function construction, this corresponds to rather different $k_{F 1}^{(0)}$ and $k_{F 1}^{(\pi)}$, with significantly more $d_{1}$ fermions in the bonding band than in the antibonding band; see Fig. 2. When we increase the ring coupling, $q_{x}^{(0)}$ increases, while $q_{x}^{(\pi)}$ decreases, corresponding to moving the $d_{1}$ fermions from the bonding to the antibonding level and making the two bands more equally populated. Interestingly, we find that the relation $q_{x}^{(0)}+q_{x}^{(\pi)}=2 \pi \rho$ is satisfied, consistent with the prediction from the gauge theory of a Luttinger-type theorem in the correlated boson phase.

The identification with the DBL is bolstered by a trial wave-function study. The optimal such state for the system in Fig. 8 is

$$
\Psi_{\text {bos }}=\left[\operatorname{det}_{1}\left(N_{1}^{(0)}=20, N_{1}^{(\pi)}=12\right)_{\mathrm{abc}}\right]^{0.75}
$$



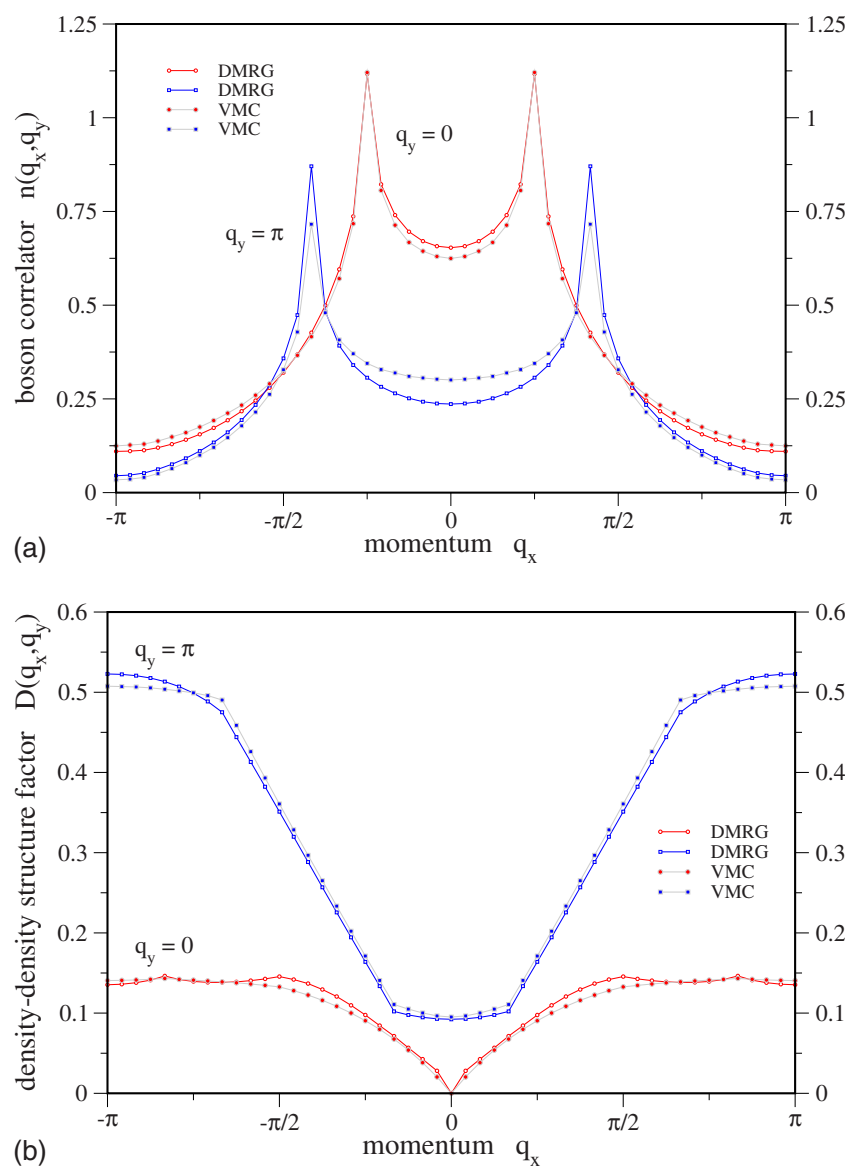

FIG. 8. (Color online) (a) The boson occupation number $n(\mathbf{q})$ and (b) the density-density structure factor $D(\mathbf{q})$ for the two-leg system at $\rho=1 / 3, J_{\perp}=J, K=3 J$; the system length is $L_{x}=48$. The results are representative of the DBL phase.

$$
\times\left[\operatorname{det}_{2}\left(N_{2}^{(0)}=32, N_{2}^{(\pi)}=0\right)_{\mathrm{abc}}\right]^{0.1} .
$$

The length of the system is $L_{x}=48$ and the total boson number is $N_{b}=32$. The $d_{1}$ determinant is constructed from $N_{1}^{(0)}$

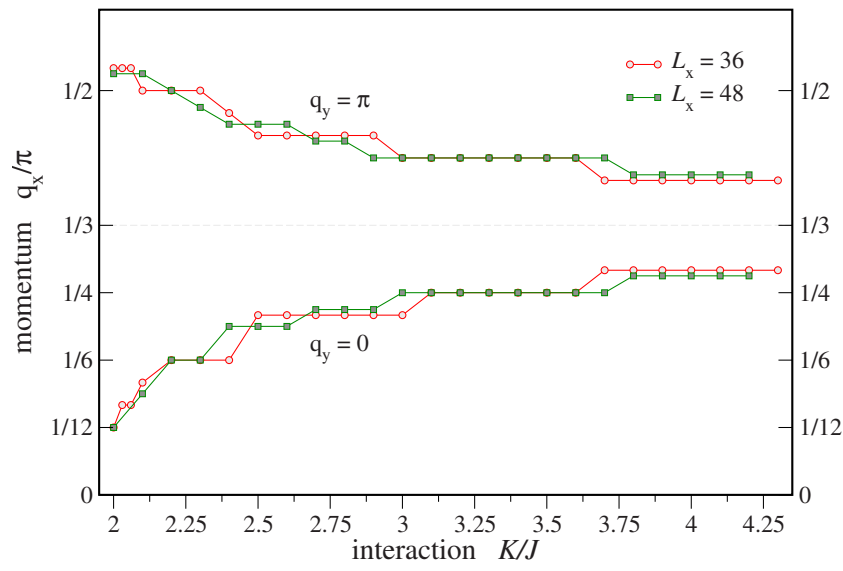

FIG. 9. (Color online) Positions of the singular momenta in the DBL phase as a function of the ring exchange $K$ for a system with $J_{\perp}=J$ and filling $\rho=1 / 3$. Data for systems with length $L_{x}=36$ and $L_{x}=48$ are given. The momenta are determined from the peak values of $n\left(q_{x}, q_{y}\right)$ calculated by DMRG such as shown in the top panel of Fig. 8 .

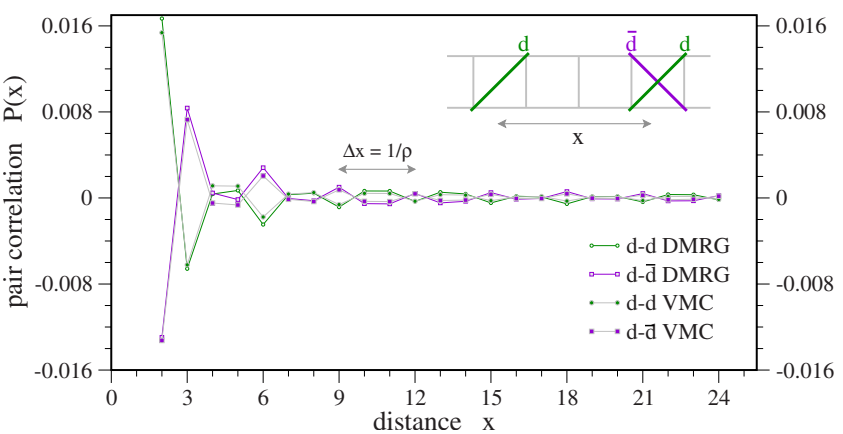

FIG. 10. (Color online) The boson pairing correlations in real space for the DBL system described in Fig. 8 calculated by DMRG and VMC. We show correlations for pairs on the diagonals $d$ and $\bar{d}$, while values for other pair orientations are much smaller.

$=20$ bonding and $N_{1}^{(\pi)}=12$ antibonding orbitals, while the $d_{2}$ determinant is constructed from bonding orbitals only. To define the orbitals, we use antiperiodic boundary conditions ("abc") along the $\hat{x}$ direction for both $d_{1}$ and $d_{2}$, which gives closed shells for the above occupation numbers, while the physical boson wave function respects periodic boundary conditions and carries zero total momentum. The determinant powers are understood as $(\text { det })^{p} \equiv \operatorname{sgn}($ det $) \mid$ det $\left.\right|^{p}$. The $d_{2}$ determinant can be written explicitly as

$$
\operatorname{det}_{2}\left[N_{2}^{(0)}=N_{b}, N_{2}^{(\pi)}=0\right] \propto \prod_{i<j} \sin \frac{\pi\left(x_{i}-x_{j}\right)}{L_{x}}
$$

and in particular prevents two bosons from sharing the same rung. In fact, the exact ground states in the ED/DMRG have very small double occupancy of rungs deep in this phase, as illustrated in the lower panel of Fig. 7. The characteristic boson wave vectors in the above DBL state are $q_{x}^{(0)}=k_{F 2}$ $-k_{F 1}^{(0)}=12 \pi / 48$ and $q_{x}^{(\pi)}=k_{F 2}-k_{F 1}^{(\pi)}=20 \pi / 48$. These bosons are created by occupying $d_{1}$ and $d_{2}$ orbitals on the opposite sides, which is motivated by the Amperean enhancement of such composites as described earlier. The overall match of the trial wave-function results with the exact DMRG correlations is striking, reproducing the singular features as illustrated in Fig. 8.

Turning to the density structure factor $D\left(q_{x}, q_{y}\right)$, there are many features but they are also weaker than in $n\left(q_{x}, q_{y}\right)$. For $q_{y}=0$, we expect a linear $\left|q_{x}\right|$ behavior near $q_{x}=0$ and signatures at $2 k_{F 1}^{(0)}=40 \pi / 48$ and $2 k_{F 1}^{(\pi)}=24 \pi / 48$, which we see, and also at $\left|2 k_{F 2}\right|=32 \pi / 48 \bmod 2 \pi$, which we do not see in either DMRG or VMC. (We can understand this in the wave function because the power for the $\operatorname{det}_{2}$ is small and suppresses density correlations coming from this piece.) At $q_{y}$ $=\pi$, we expect signatures at $k_{F 1}^{(0)}+k_{F 1}^{(\pi)}=32 \pi / 48$ and at $k_{F 1}^{(0)}$ $-k_{F 1}^{(\pi)}=8 \pi / 48$, both of which we see. As discussed in Appen$\operatorname{dix} \mathrm{A}$, it is a complicated matter to predict the exponents of the different singularities; given our limited system sizes, we do not attempt measuring these. It is remarkable that the DBL wave function can reproduce the singular features, but we do not know at this point whether it can reproduce all exponents in general.

Finally, in Fig. 10 we show the diagonal-diagonal pairing correlator. It is more short ranged than in the SF phase as 

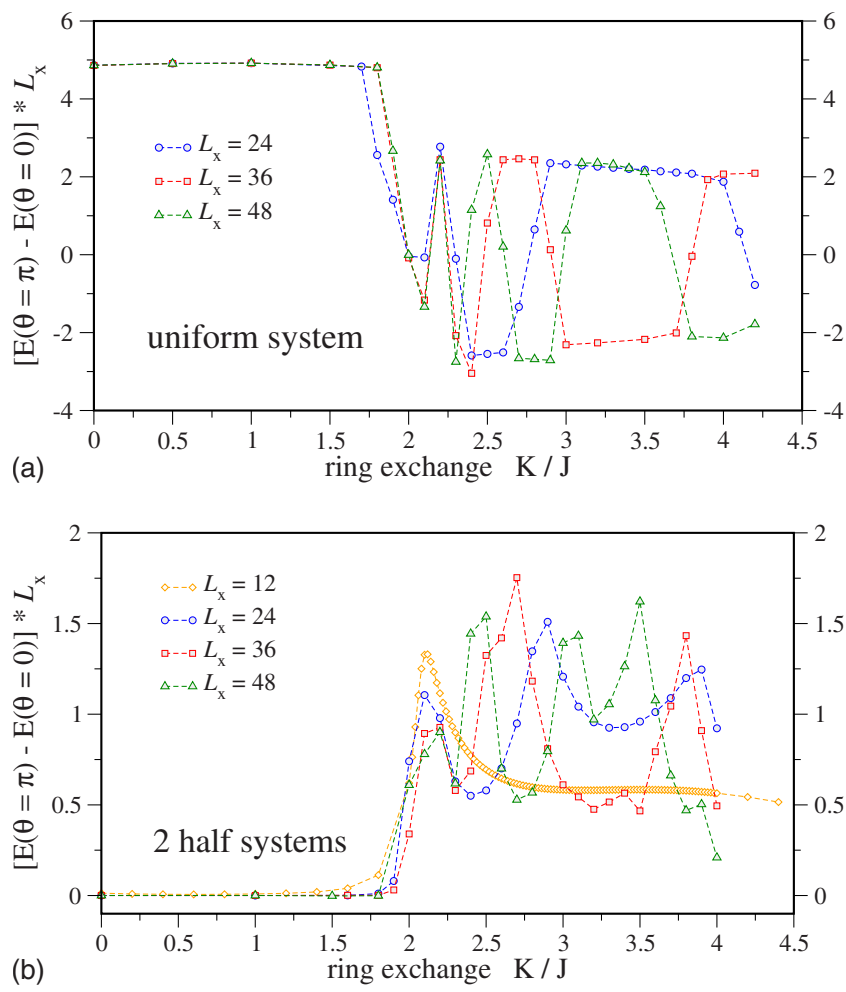

FIG. 11. (Color online) Detection of the boson propagation around the loop by applying a phase twist $\theta$ to the periodic boundary connection along the $\hat{x}$ direction. The energy differences $\Delta E$ $=E(\theta=\pi)-E(\theta=0)$, multiplied by $L_{x}$, for a uniform system with $J_{\perp}=J$ for all bonds (top panel) and for a specially designed system with $J_{\perp}=J$ in one half and $J_{\perp}=-J$ in the other half of the system (bottom panel). The calculations are done by DMRG.

well as the two paired phases described below, but is characteristic of the DBL phase. First, $P_{2 b}(x)$ has robust sign oscillations at wave vector $2 \pi \rho$. Second, the $+45^{\circ}$ and $-45^{\circ}$ diagonal pairs have opposite signs; the nontrivial sign structure in the wave function gives some $d$-wave-correlated character to the boson liquid, which is observed in the two-boson correlator.

\section{Detection of boson bonding and antibonding propagation by two stiffnesses}

In this subsection we want to further highlight some characteristic differences between the SF and the DBL phases. In the SF state, the wave function has QLRO at $\mathbf{q}=0$. Imposing a twist boundary phase $\theta$ in the boson hopping term in Hamiltonian (3), one can measure the superfluid stiffness as $\rho_{s}=\partial^{2} E / \partial \theta^{2}$, which is found to scale as $1 / L_{x}$ in the SF phase. This stiffness calculated from ED is shown for the small $L_{x}$ $=12$ system in the top panel of Fig. 7. For larger system lengths, the stiffness can alternatively be estimated by DMRG calculations of the energy difference for a $\theta=\pi$ twist, i.e., $\rho_{s} \sim \Delta E=E(\theta=\pi)-E(\theta=0)$.

In the top panel of Fig. 11, we show $L_{x} \Delta E$ as a function of the ring exchange $K / J$ for several system sizes $L_{x}$ $=24,36,48$. For isotropic hopping $J_{\perp}=J$, we find that $L_{x} \Delta E$ is essentially constant in the SF phase but drops abruptly at the SF to DBL transition. In the DBL phase, $L_{x} \Delta E$ shows a rather irregular behavior including sign changes when varying either $K$ or $L_{x}$. For a fixed system length $L_{x}$, the more abrupt changes can be matched with the steplike changes in the singular momenta positions shown, e.g., in Fig. 9 for $L_{x}=36,48$. For fixed ring exchange $K$, the abrupt changes with varying $L_{x}$ are indicative of strong incommensurate correlations, where different $L_{x}$ offer varying degrees of matching at the (twisted) periodic boundary: $\Delta E>0$ means that $\theta=0$ boundary conditions provide better matching than $\theta$ $=\pi$ does, while the situation is reversed if $\Delta E<0$.

A finite $\left|L_{x} \Delta E\right|$ in the large system limit in the above setting implies that there is a propagating "bonding" mode (i.e., with transverse momentum $q_{y}=0$ ). Based on our picture of the DBL phase, there must also be a propagating "antibonding" mode with $q_{y}=\pi$, which we would like to detect directly and contrast with the SF phase where there is no such mode.

To this end, we design a system composed of two halves connected at both ends, where the left half is a $\left(J, J_{\perp}\right.$ $=J, K)$ Hamiltonian while the right half is a $\left(J, J_{\perp}=-J, K\right)$ Hamiltonian. First, we note that a model with $J_{\perp}=-J$ can be related to a model with $J_{\perp}=J$ by a transformation $b(x, y)$ $\rightarrow(-1)^{y} b(x, y)$. This ensures that the boson densities are the same in the two parts. Crucially, this transformation interchanges the notions of bonding and antibonding modes.

If the designed system is in the regime of the regular SF phase, the gapless bonding bosons in the half with $J_{\perp}=J$ cannot penetrate into the other half, where the gapless mode is antibonding, and vice versa. Since the bosons are not able to propagate around the loop, a $\theta=\pi$ twist should not produce a significant change $\Delta E$ which will vanish exponentially with $L_{x}$. On the other hand, in the DBL phase there are gapless modes of both bonding and antibonding types in either region, so that bosons can propagate around the loop, and the $\pi$ twist should change the energy by an amount proportional to $1 / L_{x}$. The "bonding/antibonding propagation stiffness" $L_{x} \Delta E$ for the designed two-half system is shown in Fig. 11 as a function of $K / J$. Indeed, we find a vanishingly small $L_{x} \Delta E$ in the SF phase and a rather irregular but nevertheless finite $L_{x} \Delta E$ in the DBL phase. ${ }^{30}$

\section{Extended $s$-wave-paired boson phase}

For our two-leg ladder system, one might expect to find the $\operatorname{DBL}[2,2]$ phase if the interchain coupling $J_{\perp}$ is much smaller than the coupling $J$ along the chains: When the boson hopping between the chains is reduced, one expects that the fermion hopping between the chains is also reduced for both species, which in turn could bring the antibonding band of the $d_{2}$ fermions to cross the chemical potential as shown in the bottom right panel of Fig. 2. DMRG studies of the ring model at filling $\rho=1 / 3$ and small $J_{\perp} \leq 0.3$ indeed find a phase that is distinct from both the DBL[2,1] and the SF as shown in the phase diagram in Fig. 3 around $K \sim 1.4 J$. However, our measurements indicate that this phase is not DBL[2,2] but a paired phase. As shown in Fig. 12 for a system at $\rho=1 / 3$ with $J_{\perp}=0.1 J, K=1.4 J$, and length $L_{x}=48$, this phase is characterized by a quite flat region in the boson occupation number $n\left(q_{x}, q_{y}\right)$ near $q_{x}=0$ without any singular 

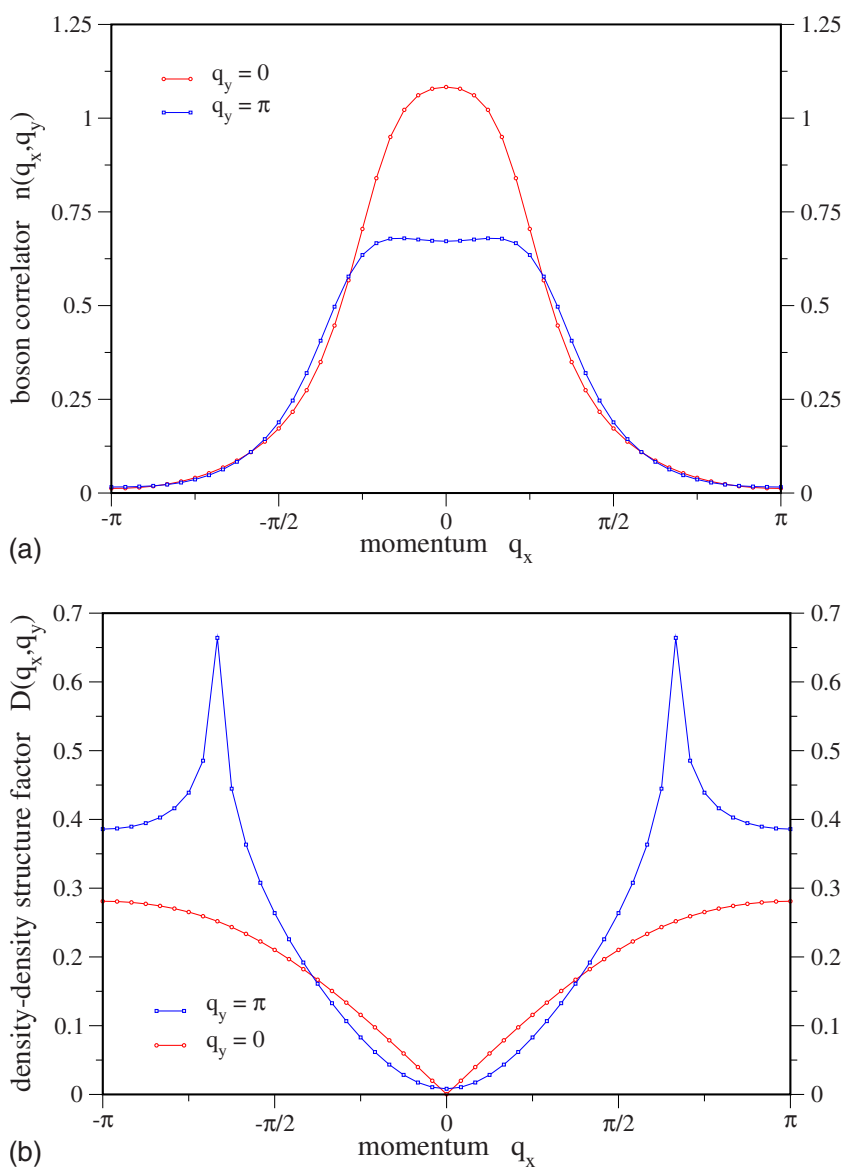

FIG. 12. (Color online) DMRG results for (a) the boson occupation number $n(\mathbf{q})$ and (b) the density-density structure factor $D(\mathbf{q})$ for a two-leg system at $\rho=1 / 3, J_{\perp}=0.1 \mathrm{~J}, K=1.4 \mathrm{~J}$; the system length is $L_{x}=48$. The results are representative for the $s$-wave-paired phase.

points, which is different from both the SF and the DBL phases. On the other hand, the density-density structure factor $D\left(q_{x}, q_{y}\right)$ has a singular behavior at a wave vector $(2 \pi \rho, \pi)$. This singular behavior can be approximated by $\sim\left|\delta q_{x}\right|^{p_{\rho}-1}$, with $p_{\rho}$ between 1.33 and 1.50 , providing the best fit to the data, which corresponds to power-law envelope $x^{-p_{\rho}}$ in real space. [Note that when $J_{\perp}=0$ the number of bosons in each chain is independently conserved and $D\left(q_{x}, \pi\right)$ must vanish at $q_{x}=0$, which explains the small value of $D\left(q_{x}\right.$ $=0, \pi)$ extracted by DMRG when $J_{\perp}=0.1 J$ in Fig. 12.]

The observed phase can be identified as an $s$-wave bosonpaired phase with QLRO pairing correlation as shown in Fig. 13. The pairing correlation $P_{2 b}(x)$ in this phase is strongly enhanced at large distances $x$ when compared to the correlation in the DBL phase. The values are roughly similar for pairs straddling the chains but smaller for pairs inside the chains. The decay of $P_{2 b}(x)$ can be fitted by a power-law

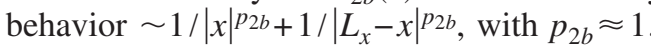

We have also checked the single-boson correlation in real space: It is much reduced compared to the nearby SF and DBL phases and decays very quickly-essentially exponentially. In particular, it is much smaller than the boson-pair correlation beyond a few lattice spacings. We then conclude that there is a gap to single-boson excitations but not to pairs.

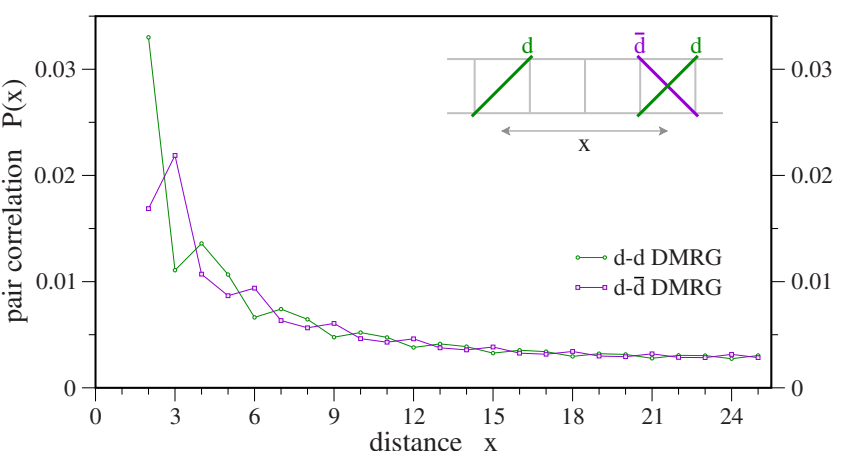

FIG. 13. (Color online) The boson pairing correlations in real space for the same $s$-wave-paired system described in Fig. 12. We show diagonal pairs but roughly similar correlations are obtained also for pairs on the rungs, while correlations for pairs in the chains are significantly smaller.

This identification of the phase is also supported by a VMC study using paired boson wave functions described in Appendix $C 2$, which improve the energetics over that of the trial DBL states and reproduce qualitatively the DMRG correlations.

Interestingly, the $s$-wave-paired phase can be regarded as an instability of the DBL[2,2] phase. In Appendix A 3, we argue that without specially adjusted short-range interactions, the $\mathrm{DBL}[2,2]$ is unstable once the gauge fluctuations are included, and the most natural outcome is a paired phase with the same phenomenology as described above. In particular, the pairs are $s$-wave, carry zero momentum, and show QLRO with power law $x^{-p_{2 b}}$, while we also predict power-law density correlations at wave vector $(2 \pi \rho, \pi)$ with $x^{-p}$. In Appendix A 3 we also predict that the two exponents are inverses of each other, $p_{2 b} p_{\rho}=1$. The DMRG estimates of the power laws differ somewhat from this, but it is hard to make them accurate given the slow decay of the correlations and our limited system sizes.

\section{D. $d$-wave boson-paired phase}

Consider now bosons at small filling $\rho=1 / 9$ (Fig. 4). As we further increase $K$, the DBL phase in the intermediate range becomes unstable and another phase emerges. As shown in Fig. 14 for a system at filling $\rho=1 / 9$ with $J_{\perp}$ $=0.1 J, K=3 J$, and length $L_{x}=54$, this phase is characterized by $n\left(q_{x}, 0\right) \approx n\left(q_{x}, \pi\right)$, which is satisfied very accurately and is indicative of strongly suppressed boson correlator between the chains. We think that the peaks in $n(\mathbf{q})$ are nonsingular and represent short-range boson correlations, while $G_{b}(\mathbf{r})$ decays exponentially on long distances: While we cannot ascertain the exponential decay with our system sizes, the single-boson correlator is already more than an order of magnitude smaller and decays much faster than the pair correlator described below. This phase is further identified as a $d$-wave boson-paired phase with power-law pairing correlation as shown in Fig. 15. The diagonal pairing correlations $P_{2 b}(x)$ show opposite signs depending on the relative orientation of the two pairs: positive when both are $+45^{\circ}$ diagonals and negative when one is $+45^{\circ}$ and the other is $-45^{\circ}$, 

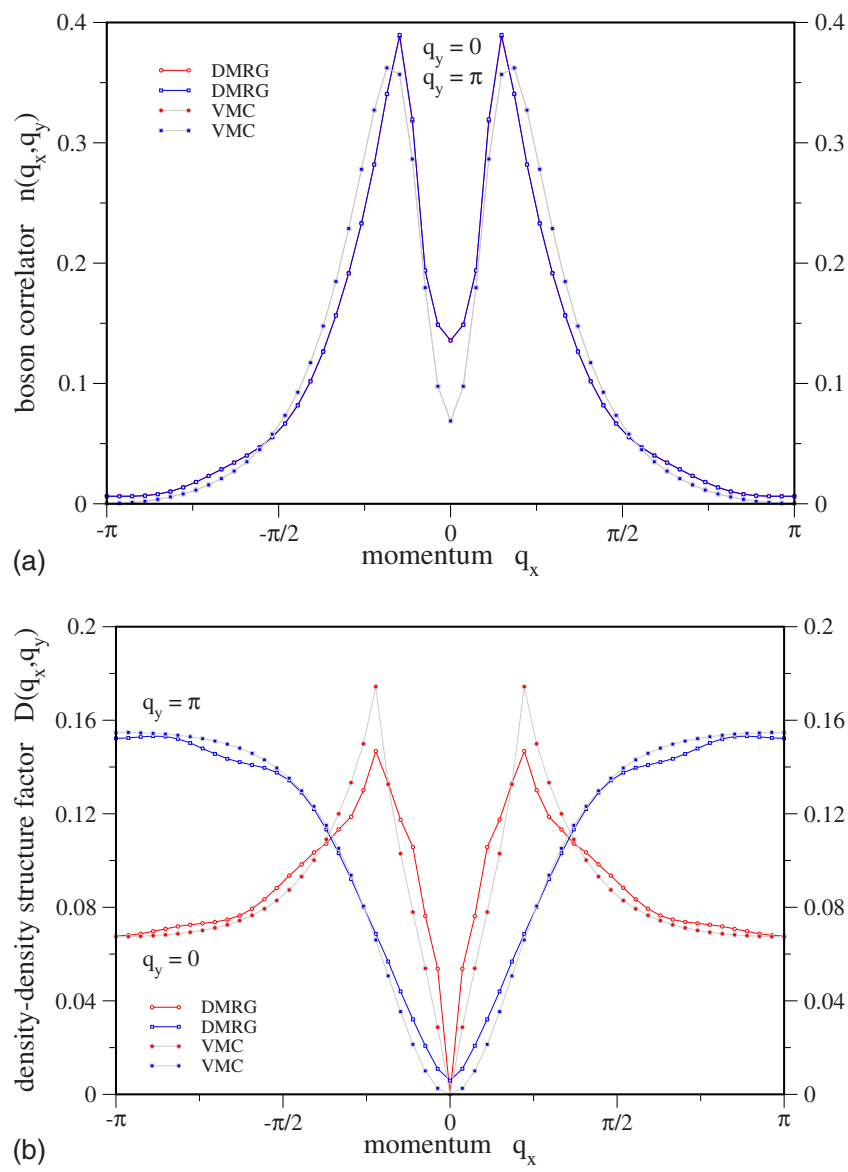

FIG. 14. (Color online) (a) The boson occupation number $n(\mathbf{q})$ and (b) the density-density structure factor $D(\mathbf{q})$ for a two-leg system at $\rho=1 / 9, J_{\perp}=0.1 J, K=3 J$; the system length is $L_{x}=54$. The results are representative of the $d$-wave-paired phase.

which is consistent with the $d$-wave-pairing picture. We find a power-law correlation $P_{2 b}(x) \sim 1 / x^{p_{2 b}}$, with an estimated exponent $p_{2 b} \approx 0.85$.

The identification of this phase as a $d$-wave-paired phase is supported by the reasoning at low densities described in Appendix B 3. Thus, for a pair of bosons on an otherwise empty ladder, there is a transition at $K / J=2$, at which it becomes favorable for bosons to form a molecule with internal $d$-wave character. If we now have a system of bosons at small density, it is natural that there will be a parameter

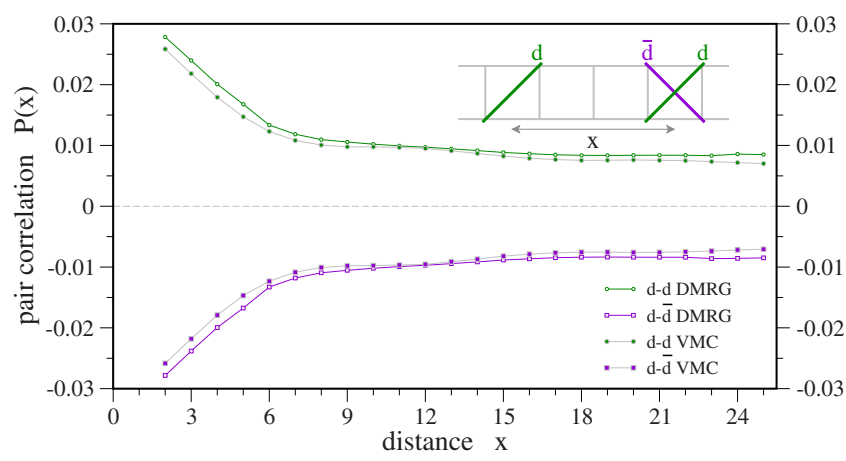

FIG. 15. (Color online) The boson pairing correlations in real space for the same system described in Fig. 14. range where the bosons are paired and the resulting molecules form a Luttinger liquid.

To bolster this identification, we also studied such states variationally. As described in Appendix C 1, we construct a trial wave function as a product of a Pfaffian and a determinant, since these are simple to work with in VMC. The optimal trial state for the system in Fig. 14 is

$$
\Psi_{\text {bos }}=\operatorname{Pf}_{1} \times\left[\operatorname{det}_{2}\left(N_{2}^{(0)}=12, N_{2}^{(\pi)}=0\right)\right]^{0.3} .
$$

The system length is $L_{x}=54$ and the boson number is $N_{b}$ 12. The Pfaffian is for an antisymmetric (spinless fermion) pair function $g\left(x, y ; x^{\prime}, y^{\prime}\right)=\delta_{y \neq y^{\prime}} \operatorname{sgn}\left(y-y^{\prime}\right) e^{-\left|x-x^{\prime}\right| \xi \xi}$ that connects only sites on different chains. The optimal "size of the pair" is $\xi=1.8$. This is a " $p_{y}$ " pairing; when composed with a " $p_{x}$ " character present in the determinant, this gives a $d$-wave character to the boson pairs. Strictly speaking, the above state is valid only in the $J_{\perp}=0$ limit, since it prevents fluctuations of the boson number in each chain. Still, as we can see from Fig. 14, the variational wave function reproduces the DMRG correlations surprisingly well in this phase. The boson momentum distribution function for the trial state is (by construction) nonsingular, so that the close agreement with the DMRG found for $L_{x}=54$ strengthens the identification of this phase as $d$-wave paired.

We also mention the signatures in the boson density shown in the lower panel of Fig. 14. $D\left(q_{x}, 0\right)$ shows the familiar $\left|q_{x}\right|$ at small momenta and also a singularity at $2 \pi \rho$. The latter gives a wavelength equal to the spacing between the pairs, which is expected in a Luttinger liquid of such molecules. The density singularity is not too strong and is in line with the slow decay of the pair correlator.

Unlike the $s$-wave case, we were not able to access the $d$-wave-paired phase from the DBL theory. More precisely, once we postulate the continuum gauge theory, the kind of analysis we can do is to consider allowed fermion interactions and see if they can become relevant and what the resulting strong-coupling phase might be. The $d$-wave-paired phase does not come up from such "weak-coupling" arguments around the continuum theory, and we only analyze it directly in the ring model at strong coupling (Appendixes B 3 and $\mathrm{C} 1$ ).

\section{E. Phase separation}

As we further increase the ring exchange toward $K \gtrsim 4 J$, the uniform phase becomes unstable to phase separation. For such large $K$, the boson density will separate into half-filled and empty regions, which is energetically favored by the ring-exchange term. In the ED of the small $L_{x}=12$ system, phase separation is identified to happen at $K \gtrsim 5 \mathrm{~J}$, where all the lowest-energy states from different momentum sectors become nearly degenerate. In the DMRG calculation, the phase separation is indicated by a nonuniform boson density in the obtained ground state, which breaks the translational symmetry due to small perturbations from the cutoff of the Hilbert space in DMRG. The phase separation occurs around $K \gtrsim 4 J$ as observed from DMRG. Finally, the phase separation can also be studied in VMC by measuring trial energies for different boson densities and performing Maxwell con- 
struction. (This is described in detail, e.g., in Sec. VII of Ref. 17 for the 2D ring model.)

The tendency toward phase separation for large $K$ is expected since the ring term provides effective attraction between bosons; see Appendix B 3 and Sec. VII of Ref. 17. Also, in the large $K$ limit, we can solve the ladder Hamiltonian exactly and find phase separation-this is shown in Appendix B 2. Nevertheless, for intermediate $K$ the various phases discussed above are stabilized by the boson hopping.

\section{CONCLUSIONS AND FUTURE DIRECTIONS}

An obvious virtue of searching for the fingerprint of putative 2D gapless quantum phases in quasi-1D models is the relative numerical and analytic tractability of the latter. An important requirement of this approach is that the 2D quantum phase has singular correlations along surfaces in momentum space. In this case the number of 1D gapless modes present on an $N$-leg ladder will necessarily scale linearly with $N$. While the particular fingerprint of the $2 \mathrm{D}$ quantum phase can already be present on the two-leg ladder, as was the case for the DBL phase studied exhaustively in this paper, a compelling approach to two dimensions will certainly require analyzing models with larger $N$.

When $N=3$ already, exact diagonalization studies become problematic since finding the ground states of boson or spin models with more than 36 sites becomes virtually impossible. Thus a three-leg ladder of length $L=12$ is already near the limit. DMRG is much more promising, and past studies on spin models reveal that convergence to the ground state is possible for lengths $L$ of the order of 50-24 with $N=3-6$, respectively.

Variational Monte Carlo for Gutzwiller-type wave functions constructed as products of determinants or Pfaffians as considered in this paper is possible for significantly larger $N$, and equal-time correlation functions can be computed with a fair degree of accuracy. However VMC is only as good as the variational wave function being employed, and the quality of the wave function is hard to assess in the absence of other more accurate methods. The ground-state energy of all physically reasonable Hamiltonians is sensitive almost entirely to very short-range correlations, whereas for gapless quantum systems it is usually the longer-range correlations which are necessary in order to distinguish between various competing phases. Quantum states in two dimensions with gapless critical surfaces in momentum space are somewhat of an exception since the location of such surfaces is presumably due to short-range correlations in the signs and amplitudes of the wave function. However the precise singular structure on such surfaces reflects longer-range correlations in the wave function.

Gauge theory ${ }^{1}$ seems to offer one of the few analytic approaches to access putative gapless spin-liquid-type quantum phases in two dimensions that have no quasiparticle description. Moreover for mean-field states with a spinon Fermi sea, a controlled inclusion of the gauge fluctuations is problematic, even in the simplest case of a compact U(1) gauge field. Accounting for the instanton (monopole) events which reflect the compact nature of the gauge field is exceedingly difficult. Even for a noncompact U(1) gauge field coupled to a Fermi sea, while there does exist a random-phase approximation (RPA)-type approach, ${ }^{1,11-13}$ it involves uncontrolled and worrisome approximations.

However for the ladder systems, gapless fermions (spinons) coupled to a U(1) gauge field is eminently tractable, although this fact has been exploited rather infrequently. ${ }^{19-22}$ In one dimension the gauge field has no transverse component and the bosonization method can be employed, leading to a Gaussian effective-field theory for the low-energy excitations. This is another distinctive advantage of studying ladder systems relative to their 2D counterparts.

\section{A. DBL phase on the $N$-leg ladder}

One extension of this paper involves studying the boson ring Hamiltonian on ladders with $N=3,4,5$, and larger. Already for $N=3$ there are a number of additional features that are not present on the two-leg ladder. First, one can consider either periodic or antiperiodic boundary conditions for the bosons in the rung direction. It is quite plausible that the phase diagram and the presence/absence of the DBL phase will depend on this choice. Second, while our search for a DBL phase with three gapless modes on the two-leg ladder was unsuccessful due to instabilities of the $\mathrm{DBL}[2,2]$ variational state, it seems likely that for $N \geq 3$ a DBL phase with more gapless modes than $N$ should be accessible. This is interesting since in such a case at least two of the 1D modes would have the same transverse momentum.

For a general $N$-leg ladder one can construct variational states of the form $\operatorname{DBL}[n, m]$ with integer $n, m$ satisfying $N$ $\geq n \geq m>0$ (with $n, m$ as the number of partially filled bands for the $d_{1}, d_{2}$ partons, respectively). The number of 1D gapless modes in such a phase is $n+m-1$, so that $\mathrm{DBL}[3,2]$ for $N=3$, say, would have four 1D modes. If $m=1$ the $d_{2}$ fermion has only one band partially occupied. Assuming the band has $k_{y}=0$, the corresponding variational wave function vanishes whenever two or more bosons occupy a rung. In this case of no-double rung occupancy, the determinant for the $d_{2}$ fermion can be viewed as a (strictly) 1D Jordan-Wigner string multiplying the $d_{1}$ determinant. Whenever $m>1$ this is no longer the case, and the $d_{2}$ determinant would have a more subtle effect on the signs of the boson wave function. It would thus be desirable to access a DBL phase with $m>1$. As detailed in Appendix A, the gauge theory suggests an instability of the DBL[2,2] state for $N=2$ due to a rather special "nesting" condition present for $n=m=2$. For $n>m$ $\geq 2$ this will not generally be the case so we expect that such DBL phases should be more easily accessible for $N=3,4, \ldots$. It would also be interesting to use the DMRG to access some dynamical information about the DBL, which should be possible at least for $N=2,3$. For $N \rightarrow \infty$ the ladder model recovers the full symmetry of the $2 \mathrm{D}$ square lattice, and in future work it would be desirable to study how to approach this limit.

\section{B. SU(2)-invariant spin models with ring exchange}

It would be most interesting to search for possible 2D spin-liquid phases with singular surfaces in momentum space 
in models possessing SU(2) spin symmetry. Recently, several authors ${ }^{15,16,18}$ suggested that the putative spin liquid observed in $\kappa-(\mathrm{ET})_{2} \mathrm{Cu}_{2}(\mathrm{CN})_{3}$ is of such character. They proposed that a mean-field state with a Fermi surface of spinons is an appropriate starting point. Variational energetics on the corresponding Gutzwiller projected spinon Fermi sea have been performed on the triangular lattice Heisenberg antiferromagnet with a four-site cyclic ring-exchange term, a model argued to be relevant for a Mott insulator such as $\kappa-(\mathrm{ET})_{2} \mathrm{Cu}_{2}(\mathrm{CN})_{3}$ that has a comparatively small charge gap.

It should be possible to study the Heisenberg plus ringexchange spin Hamiltonian on the triangular strip to search for signatures of the proposed parent 2D spin liquid. In the absence of ring exchange, this model is equivalent to the 1D Heisenberg model with both first- and second-neighbor interactions $J_{1}$ and $J_{2}$; it was explored using DMRG in earlier work. ${ }^{31,32}$ The phase diagram as a function of $J_{2} / J_{1}$ appears to have two phases: a Bethe-chain phase stable for small $J_{2} / J_{1}$ with one gapless mode and a fully gapped dimerized state for intermediate and large $J_{2} / J_{1}$. The gap decreases exponentially for large $J_{2} / J_{1}$, where we have nearly decoupled legs $\left(J_{2}\right)$ of the triangular ladder (with interchain $J_{1}$ ).

With a four-site ring-exchange term (coupling $K$ ), the triangular strip model is characterized by two dimensionless parameters, $J_{2} / J_{1}$ and $K / J_{1}$. Are there any additional phases in this phase diagram? The spin-liquid phase on the triangular strip that descends from the (putative) 2D spinon Fermisurface state should have three gapless modes (two for spin, two for the two legs, and minus one from the gauge constraint) and corresponding incommensurate spin correlations. Variational Monte Carlo together with a gauge theory analysis can provide a detailed characterization of this state, which could then be compared with DMRG, precisely as was done in this paper for the boson ring model. If present, one expects the spin-liquid phase to appear for intermediate values of both $J_{2} / J_{1}$ and $K / J_{1}$.

In fact, Klironomos et al. ${ }^{33}$ considered such a $J_{1}-J_{2}-K$ model, motivated by the study of Wigner crystals in a quantum wire using ED of systems up to $L=24$. They found an unusual phase in this intermediate regime but it had proven difficult to clarify its nature. We conjecture that this may contain the spin-liquid phase but further studies are warranted.

In addition, one might study a half-filled Hubbard model on the triangular strip, which should exhibit a metal-insulator transition at intermediate coupling $U / t$. Of particular interest is the nature of the quantum state just on the insulating side of the Mott transition. If the Mott transition is weakly first order, there will be substantial charge fluctuations and ringexchange interactions in this part of the phase diagram and possibly a spin-liquid phase, in addition to phases that have already been identified (see Refs. 34-36 and references therein).

\section{Itinerant electrons}

What are the prospects of using ladders to approach nonFermi-liquid phases of 2D itinerant electrons? One complication is as follows: Imagine a weak-coupling Hubbard model on the square lattice (at densities well away from special commensurate values) which has a conventional 2D Fermi-liquid ground state. On an $N$-leg ladder, the free Fermi points present for $U=0$ would be converted into Luttinger liquids. The jump discontinuity in the momentum distribution function at each Fermi point would be lost, but singularities would remain, characterized by some Luttinger liquid exponents. Moreover, the location of the singularities would still satisfy the Luttinger sum rule. Now imagine some strong-coupling 2D electron Hamiltonian with a non-Fermiliquid phase that has a residual Luttinger Fermi surface but with $Z=0$, analogous to a $1 \mathrm{D}$ Luttinger liquid. The corresponding $N$-leg ladder descendant would be qualitatively indistinguishable from the phase of the weak-coupling Hamiltonian. Conversely, the presence of a Luttinger-satisfying Fermi surface on an $N$-leg ladder would not enable one to distinguish between the two different 2D phases, one a Fermi liquid and the other not.

On the other hand, the $N$-leg ladder descendant of a $2 \mathrm{D}$ non-Fermi-liquid ground state with momentum space singularities that violate the Luttinger sum rule (surfaces with the "wrong" volume or perhaps even arcs) would have qualitatively distinct signatures. Several recent papers ${ }^{17,18}$ proposed such 2D non-Fermi-liquid phases, and it would be extremely interesting to find evidence for their ladder descendants. The wave function for one of these phases was constructed ${ }^{17}$ by taking the product of a free fermion determinant and the DBL wave function in Eq. (2). This phase, which inherits the $d$-wave sign structure from the bosonic DBL wave function, was called a $d$-wave metal and would exhibit distinctive signatures if present on a ladder system. A possible Hamiltonian that might possess the $d$-wave metal can be expressed by adding an "itinerant-electron-ring" term to the usual square lattice $t$ - $J$ model: $H=H_{t-J}+H_{\text {ring }}^{\text {el }}$, with

$$
H_{\text {ring }}^{\mathrm{el}}=K_{\mathrm{el}} \sum_{\square}\left(\mathcal{S}_{1,3}^{\dagger} \mathcal{S}_{2,4}+\text { H. c. }\right),
$$

where sites $1,2,3$, and 4 are taken to run clockwise around elementary square plaquettes and the summation runs over all plaquettes. Here we have defined an electron singlet creation operator:

$$
\mathcal{S}_{i, j}=\left(c_{i \uparrow}^{\dagger} c_{j \downarrow}^{\dagger}-c_{i \downarrow}^{\dagger} c_{j \uparrow}^{\dagger}\right) / \sqrt{2} .
$$

The ring term rotates a singlet on the 2-4 diagonal into a singlet on the 1-3 diagonal. It would be interesting to study this or other such strong-coupling Hamiltonians on the $\mathrm{N}$-leg ladder.

\section{Conclusions}

To conclude, in this paper we have initiated a study of $N$-leg ladder systems that are descendants of candidate 2D quantum phases with low-energy excitations residing on singular surfaces. Motivated by one such proposal for the socalled DBL phases of uncondensed itinerant bosons, ${ }^{17}$ we have studied the two-leg model with frustrating ring exchanges using exact numerical approaches. We have indeed found the DBL[2,1] ladder version arising prominently in the phase diagram. We have also searched for the DBL[2,2] ver- 
sion but from a gauge theory analysis concluded that it is likely unstable due to special kinematics on the two-leg ladder and instead gives rise to a boson-paired phase with extended $s$-wave character in the pairs. This paired phase is in fact realized in our model, while the DBL theory gives us tools to understand its properties. While our focus has been on the DBL ideas, we have explored the full phase diagram of the specific ring model in fair detail and found also other strong-coupling phases such as the above $s$-wave-paired phase and the $d$-wave-paired phase. The latter is not directly accessible from the DBL theory but is characteristic of the binding tendencies in our ring terms, which eventually cause the bosons to phase separate for large ring exchanges. We hope to pursue similar ideas for $N \geq 3$ ladders and also for $\mathrm{SU}(2)$ spin and itinerant-electronic models with ring exchanges, which are particularly exciting.

\section{ACKNOWLEDGMENTS}

We would like to thank L. Balents, T. Senthil, and A. Vishwanath for useful discussions. This work was supported by DOE Grant No. DE-FG02-06ER46305 (D.N.S.), the National Science Foundation through Grants No. DMR0605696 (D.N.S.) and No. DMR-0529399 (MPAF), and the A. P. Sloan Foundation (O.I.M.). Some of our numerical simulations were based on the ALPS libraries. ${ }^{37}$

\section{APPENDIX A: GAUGE THEORY DESCRIPTION AND SOLUTION BY BOSONIZATION}

A faithful formulation of the physical system in the slaveparticle approach [Eq. (1)] is a compact U(1) lattice gauge theory. We set this up on the two-leg ladder as follows: Denote the vector potential components on the links of the two chains as $a_{\mathrm{I}, x}$ and $a_{\mathrm{II}, x}$ and on the rungs as $a_{y}$. In the Euclidean path integral, denote the temporal components associated with the sites on the two chains as $a_{\mathrm{I}, \tau}$ and $a_{\mathrm{II}, \tau}$. The action for the gauge field contains $-\cos \left(a_{\mathrm{I}, x}-a_{\mathrm{II}, x}+\nabla_{x} a_{y}\right),-\cos \left(a_{\mathrm{I}, \tau}\right.$ $\left.-a_{\mathrm{II}, \tau}+\nabla_{\tau} a_{y}\right)$, and $-\sum_{l=\mathrm{I}, \mathrm{II}} \cos \left(\nabla_{\tau} a_{l, x}-\nabla_{x} a_{l, \tau}\right)$. For each $l$ $=\mathrm{I}$, II, the last term gives a $1+1 D$ gauge action. If the cosine is interpreted as a Villain cosine, we can replace it with $\left(\nabla_{\tau} a_{l, x}-\nabla_{x} a_{l, \tau}\right)^{2}$ and treat $a_{l}$ as noncompact with no change in the results. Choosing the gauge $a_{y}=0$, the combination $a_{\mathrm{I}}-a_{\mathrm{II}}$ is massive, while the combination $a_{\mathrm{I}}+a_{\mathrm{II}}$ is massless.

Having described the gauge sector, we now consider the fermions $d_{1}$ and $d_{2}$ with the mean-field bands as in Fig. 2 and take the continuum limit using the bonding $d_{\alpha}^{(0)}$ and antibonding $d_{\alpha}^{(\pi)}$ fields near the corresponding Fermi points $P$ $=R / L=+/-$. These fields couple to $a=\left(a_{\mathrm{I}}+a_{\mathrm{II}}\right) / 2$ as to the usual gauge field, while the massive combination $a_{\mathrm{I}}-a_{\mathrm{II}}$ can be integrated out. The continuum Hamiltonian density is

$$
\begin{gathered}
h=h_{\text {kinetic }}+h_{4 \text { ferm }}, \\
h_{\text {kinetic }}=\sum_{\alpha, k_{y}, P} P v_{F \alpha}^{\left(k_{y}\right)} d_{\alpha P}^{\left(k_{y}\right) \dagger}\left(-i \partial_{x}-e_{\alpha} a_{x}\right) d_{\alpha P}^{\left(k_{y}\right)},
\end{gathered}
$$

where $e_{1}=1$ and $e_{2}=-1$ are the gauge charges of $d_{1}$ and $d_{2}$, respectively, and $v_{F \alpha}^{\left(k_{y}\right)}$ are the appropriate Fermi velocities. In the DBL[2,2] case, there are both bonding and antibonding fields present for each species $d_{1}$ and $d_{2}$. The Fermi momenta satisfy $k_{F \alpha}^{(0)}+k_{F \alpha}^{(\pi)}=2 \pi \rho$, where $\rho$ is the original boson density per site. In the DBL[2,1] case, the $d_{2}$ fermions have only bonding fields. Ultimately, it is a question of detailed energetics whether a given mean field and gauge fluctuations theory is an appropriate starting point and what the effective parameters are. For example, we can take trial wave-function calculations to justify the particular DBL and determine the Fermi wave vectors. We could also estimate the parton velocities, but given the uncertainties in particular with many interactions, we leave these as parameters in the theory.

The allowed interactions contain general density-density terms,

$$
h_{4 \mathrm{ferm}}^{(\mathrm{I})}=\sum_{b, b^{\prime}, P}\left[B_{b, b^{\prime}} \rho_{b P} \rho_{b^{\prime} P}+C_{b, b^{\prime}} \rho_{b P} \rho_{b^{\prime}-P}\right],
$$

where $b, b^{\prime}$ sum over all bands of all species, and also the following terms,

$$
\begin{aligned}
& h_{4 \mathrm{ferm}}^{(\mathrm{II})}=\sum_{\alpha} E_{\alpha}\left[d_{\alpha R}^{(0) \dagger} d_{\alpha L}^{(0) \dagger} d_{\alpha R}^{(\pi)} d_{\alpha L}^{(\pi)}+\text { H . c. }\right] \\
& +F\left[\sum_{P} d_{1 P}^{(0) \dagger} d_{2-P}^{(0) \dagger} d_{1-P}^{(\pi)} d_{2 P}^{(\pi)}+\mathrm{H} . \mathrm{c} .\right] \\
& +G\left[\sum_{P} d_{1 P}^{(0) \dagger} d_{2-P}^{(\pi) \dagger} d_{1-P}^{(\pi)} d_{2 P}^{(0)}+\mathrm{H} . \mathrm{c} .\right] .
\end{aligned}
$$

In the DBL[2,1] case, all terms that contain $d_{2}^{(\pi)}$ are absent.

The above QED2-like theory can be analyzed perturbatively in the matter-gauge coupling, e.g., in a systematic $1 / N$ expansion, as was done in Ref. 19 for the spinon-gauge treatment of the 1D Heisenberg spin chain. From such studies, we often use the following Amperean rule of thumb: Gauge interactions modify in a singular way processes that involve fermion fields with oppositely oriented group velocities. If the corresponding gauge currents are parallel (antiparallel), such processes are enhanced (suppressed), which originates from the attraction (repulsion) of currents in electromagnetism. As an example, $d_{1 R}^{\dagger} d_{2 L}^{\dagger}$ has oppositely charged particles moving in opposite directions and producing parallel gauge currents, so this bilinear is expected to be enhanced compared to the mean field. We will see this explicitly in a solution of the 1D gauge theory by bosonization, ${ }^{19,20}$ which we pursue instead of the perturbative treatment. We also caution here and will see below that the different Fermi velocities and the general allowed density-density interactions complicate the analysis significantly and can independently affect power laws, in addition to the above Amperean rule for the gauge interaction effects.

To bosonize, ${ }^{38-40}$ we write

$$
d_{\alpha P}^{\left(k_{y}\right)}=\eta_{\alpha}^{\left(k_{y}\right)} \exp \left[i\left(\phi_{\alpha}^{\left(k_{y}\right)}+P \theta_{\alpha}^{\left(k_{y}\right)}\right)\right] .
$$

Here $\phi_{\alpha}^{\left(k_{y}\right)}$ and $\theta_{\alpha}^{\left(k_{y}\right)}$ are the conjugate phase and phonon fields for each band, while $\eta_{\alpha}^{\left(k_{y}\right)}$ are Klein factors, which we take to be Hermitian operators that commute with the bosonic fields and anticommute among themselves. The kinetic Hamiltonian density becomes 


$$
h_{\text {kinetic }}=\sum_{\alpha, k_{y}} \frac{v_{F \alpha}^{\left(k_{y}\right)}}{2 \pi}\left[\left(\partial_{x} \phi_{\alpha}^{\left(k_{y}\right)}-e_{\alpha} a_{x}\right)^{2}+\left(\partial_{x} \theta_{\alpha}^{\left(k_{y}\right)}\right)^{2}\right] .
$$

The density-density interactions [Eq. (A3)] lead to generic terms of the form $\left(\partial_{x} \phi_{b}-e_{b} a_{x}\right)\left(\partial_{x} \phi_{b^{\prime}}-e_{b^{\prime}} a_{x}\right)$ and $\partial_{x} \theta_{b} \partial_{x} \theta_{b^{\prime}}$, which are strictly marginal and affect power laws. The other interactions [Eq. (A4)] produce cosines and are written separately for $\mathrm{DBL}[2,1]$ and $\mathrm{DBL}[2,2]$ below.

We proceed in the Euclidean path integral, choose the gauge $a_{x}=0$ and integrate out $a_{\tau}$. This renders the field $\theta_{c}$ $\sim \Sigma_{\alpha, k_{y}} e_{\alpha} \theta_{\alpha}^{\left(k_{y}\right)}$ massive and essentially pins it to zero. This is the suppression of the charge fluctuations in the gauge theory and realizes the microscopic constraints $d_{1}^{\dagger} d_{1}=d_{2}^{\dagger} d_{2}$ at long wavelengths. We then have two gapless modes left in DBL[2,1] and three modes in DBL[2,2] if we can assume that the cosines corresponding to $h_{4 \mathrm{ferm}}^{\text {(II) }}$ are irrelevant (see below).

To characterize the phases of the original hard-core bosons, we examine the single-boson correlations, the boson density and current correlations, and the pair-boson correlations. The microscopic boson field is written as

$$
\begin{aligned}
b(x, y) & =d_{1}(x, y) d_{2}(x, y) \\
& =\sum_{k_{1 y}, k_{2 y}, P_{1}, P_{2}} e^{i\left(P_{1} k_{F 1}^{\left(k_{1 y}\right)}+P_{2} k_{F 2}^{\left(k_{2 y}\right)}\right) x} e^{i\left(k_{1 y}+k_{2 y}\right) y} d_{1 P_{1}}^{\left(k_{1 y}\right)} d_{2 P_{2}}^{\left(k_{2 y}\right)},
\end{aligned}
$$

which we then express in terms of the continuum bosonized fields (see separate treatments of $\mathrm{DBL}[2,1]$ and $\mathrm{DBL}[2,2]$ below). For oppositely moving $P_{1}$ and $P_{2}$, the $\theta$ part has a nonzero projection onto $\theta_{c}$. Gapping out the $\theta_{c}$ by the gauge fluctuations enhances the corresponding contribution, in agreement with the Amperean rule applied to the oppositely charged $d_{1}$ and $d_{2}$.

The fermion densities are

$$
\begin{aligned}
\rho_{d_{\alpha}}(x, y)= & d_{\alpha}^{\dagger}(x, y) d_{\alpha}(x, y)=\sum_{P, P^{\prime}, k_{y}} d_{\alpha P}^{\left(k_{y}\right) \dagger} d_{\alpha P^{\prime}}^{\left(k_{y}\right)} e^{-i\left(P-P^{\prime}\right) k_{F \alpha}^{\left(k_{y}\right)} x} \\
& +e^{i \pi y} \sum_{P, P^{\prime}}\left(d_{\alpha P}^{(0) \dagger} d_{\alpha P^{\prime}}^{(\pi)}+d_{\alpha-P^{\prime}}^{(\pi) \dagger} d_{\alpha-P}^{(0)}\right) e^{-i\left(P k_{F \alpha}^{(0)}-P^{\prime} k_{F \alpha}^{(\pi)}\right) x}
\end{aligned}
$$

The two lines in the expansion separate the $q_{y}=0$ and $q_{y}$ $=\pi$ parts, and all contributions with the same nonzero momentum are grouped together (assuming generic distinct bonding and antibonding bands). We also consider the particle current on the rungs (the definition below uses the gauge choice $a_{y}=0$ made early on):

$$
\begin{aligned}
j_{\perp, \alpha}(x) & =\frac{i}{2}\left[d_{\alpha}^{\dagger}(x, 1) d_{\alpha}(x, 2)-d_{\alpha}^{\dagger}(x, 2) d_{\alpha}(x, 1)\right] \\
& =i \sum_{P, P^{\prime}}\left(d_{\alpha P}^{(0) \dagger} d_{\alpha P^{\prime}}^{(\pi)}-d_{\alpha-P^{\prime}}^{(\pi) \dagger} d_{\alpha-P}^{(0)}\right) e^{-i\left(P k_{F \alpha}^{(0)}-P^{\prime} k_{F \alpha}^{(\pi)}\right) x} .
\end{aligned}
$$

The continuum expansion resembles that for the density at $q_{y}=\pi$ but with opposite signs between $d_{\alpha P}^{(0) \dagger} d_{\alpha P^{\prime}}^{(\pi)}$ and $d_{\alpha-P^{\prime}}^{(\pi) \dagger} d_{\alpha-P}^{(0)}$. This will help to distinguish different phases in the analysis of instabilities (Appendixes A 2 and A 3). Bosonized expressions for the contributing bilinears will be given separately in the $\mathrm{DBL}[2,1]$ and $\mathrm{DBL}[2,2]$ cases. Here we only note that when the constituent particle and hole are on the opposite sides, we again have nonzero projection onto $\theta_{c}$, so the corresponding contribution is enhanced by the gauge fluctuations, in agreement with the Amperean rule.

Finally, we consider the boson-pair operator $b\left(x_{1}, y_{1}\right) b\left(x_{2}, y_{2}\right)$ and expand in terms of the continuum fermion fields. Assuming $x_{1}$ and $x_{2}$ are nearby, we characterize the pair by its center-of-mass coordinate $X=\left(x_{1}+x_{2}\right) / 2$ along the chains (this becomes the argument of the continuum fields) and also by the internal pair structure. To this end, we collect different microscopic contributions that give rise to each term $d_{1 a}(X) d_{1 b}(X) d_{2 c}(X) d_{2 d}(X)$, where $a, b, c, d$ are combined band and Fermi point indices. Using short hands $D_{1 a}(\mathbf{r})=d_{1 a} \exp \left(i \mathbf{k}_{1 a} \cdot \mathbf{r}\right)$, etc., then $b\left(x_{1}, y_{1}\right) b\left(x_{2}, y_{2}\right)$ contains

$$
\begin{aligned}
- & {\left[D_{1 a}\left(x_{1}, y_{1}\right) D_{1 b}\left(x_{2}, y_{2}\right)-D_{1 a}\left(x_{2}, y_{2}\right) D_{1 b}\left(x_{1}, y_{1}\right)\right] } \\
& \times\left[D_{2 c}\left(x_{1}, y_{1}\right) D_{2 d}\left(x_{2}, y_{2}\right)-D_{2 c}\left(x_{2}, y_{2}\right) D_{2 d}\left(x_{1}, y_{1}\right)\right] \\
= & d_{1 a}(X) d_{1 b}(X) d_{2 c}(X) d_{2 d}(X) e^{i\left(k_{1 a x}+k_{1 b x}+k_{2 c x}+k_{2 d x}\right) X} \\
& \times \Phi_{1 a, 1 b, 2 c, 2 d}\left(y_{1}, y_{2}, \xi\right),
\end{aligned}
$$

where $\xi=x_{1}-x_{2}$ is the relative coordinate along the chains. The "pair wave function" is

$$
\begin{aligned}
\Phi_{1 a, 1 b, 2 c, 2 d}\left(y_{1}, y_{2}, \xi\right)= & e^{i\left(k_{1 a y}+k_{1 b y}+k_{2 c y}+k_{2 d y}\right) Y} \\
& \times 4 \sin \left(\frac{\mathbf{k}_{1 a}-\mathbf{k}_{1 b}}{2} \rho\right) \sin \left(\frac{\mathbf{k}_{2 c}-\mathbf{k}_{2 d}}{2} \rho\right),
\end{aligned}
$$

with $Y=\frac{y_{1}+y_{2}}{2}$ and $\rho=\left(\xi, y_{1}-y_{2}\right)$. In the above, we have chosen to characterize the pair by the momentum along the $x$ axis but not by the momentum along the $y$ axis. On the two-leg ladder, it is easier to visualize pairs by keeping track of both $y_{1}$ and $y_{2}$.

Here are a few more words about this characterization on the two-leg ladder. Consider first a 2D setting, where we would write a contribution to a boson pair $b\left(\mathbf{r}_{1}\right) b\left(\mathbf{r}_{2}\right)$ by, say,

$$
\hat{O}(\mathbf{R}) e^{i \mathbf{q} \cdot \mathbf{R}} \Phi(\rho),
$$

where $\hat{O}$ is some slowly varying operator of the center-ofmass coordinate $\mathbf{R}$, while $\Phi(\rho)$ is the internal pair function in the relative coordinate $\rho$. Specializing to a square lattice, we could then distinguish $s$-wave and $d$-wave (more precisely, $\left.d_{x y}\right)$ pairing by looking at the signs of $\Phi(\rho=\hat{x} \pm \hat{y})$. On the two-leg ladder, however, separating out the transverse momentum $q_{y}$ as in two dimensions mixes things a little. Specifically, a rotation of an $\hat{x}+\hat{y}$ pair into an $\hat{x}-\hat{y}$ pair can be also achieved by a "translation" $y \rightarrow y+1 \bmod 2$. To avoid this, we instead define the pair function by writing

$$
\hat{O}_{2 \operatorname{leg}}(X) e^{i q_{x} X} \Phi_{2 \operatorname{leg}}\left(\xi, y_{1}, y_{2}\right),
$$

where $X$ and $\xi$ are the $x$ components of $\mathbf{R}$ and $\boldsymbol{\rho}$, respectively. We then characterize the pair wave function $\Phi_{2 \text { leg }}$ by the symmetry under interchanging the two legs of the ladder $\left(y_{1,2} \rightarrow y_{1,2}+1 \bmod 2\right)$. When $\Phi_{2 \text { leg }}$ is even, we call it $s$-wave, 
since then the amplitudes for a pair sitting on a $+45^{\circ}$ diagonal and a pair on a $-45^{\circ}$ diagonal are the same. When $\Phi_{2 \text { leg }}$ is odd, we call it $d$-wave, since then the amplitudes for the two diagonals are opposite. The names "s-wave" and " $d$-wave" are used in anticipation that such local pictures of the pairs on the plaquettes are the ones that survive when we build toward 2D. Returning to the DBL construction [Eq. (A12)], we get $s$-wave or $d$-wave character depending whether $k_{1 a y}+k_{1 b y}+k_{2 c y}+k_{2 d y}$ is an even or an odd multiple of $\pi$. We now specialize to the $\operatorname{DBL}[2,1]$ and $\operatorname{DBL}[2,2]$ cases in turn.

\section{DBL[2,1]}

Upon bosonization, we start with the three modes $\left(\phi_{1}^{(0)}, \theta_{1}^{(0)}\right),\left(\phi_{1}^{(\pi)}, \theta_{1}^{(\pi)}\right)$, and $\left(\phi_{2}, \theta_{2}\right)$ (we drop the bonding band label for the $d_{2}$ fermions). To proceed formally, we change to new canonical variables via

$$
\begin{gathered}
\phi_{\rho}=\frac{1}{2}\left(\phi_{1}^{(0)}+\phi_{1}^{(\pi)}+2 \phi_{2}\right), \quad \theta_{\rho}=\frac{1}{3}\left(\theta_{1}^{(0)}+\theta_{1}^{(\pi)}+2 \theta_{2}\right), \\
\phi_{c}=\frac{1}{2}\left(\phi_{1}^{(0)}+\phi_{1}^{(\pi)}-\phi_{2}\right), \quad \theta_{c}=\frac{2}{3}\left(\theta_{1}^{(0)}+\theta_{1}^{(\pi)}-\theta_{2}\right), \\
\phi_{-}=\frac{1}{2}\left(\phi_{1}^{(0)}-\phi_{1}^{(\pi)}\right), \quad \theta_{-}=\theta_{1}^{(0)}-\theta_{1}^{(\pi)} .
\end{gathered}
$$

The microscopic boson field $b(\mathbf{r})$ has the following contributions listed by their momentum [cf. Eq. (A9)]:

$$
\begin{gathered}
\left(k_{F 1}^{\left(k_{y}\right)}+k_{F 2}, k_{y}\right): d_{1 R}^{\left(k_{y}\right)} d_{2 R} \sim e^{i\left(\phi_{\rho} \pm \phi_{-}\right)} e^{i\left[(3 / 2) \theta_{\rho} \pm(1 / 2) \theta_{-}\right]} ; \\
\left(k_{F 1}^{\left(k_{y}\right)}-k_{F 2}, k_{y}\right): d_{1 R}^{\left(k_{y}\right)} d_{2 L} \sim e^{i\left(\phi_{\rho} \pm \phi_{-}\right)} e^{i\left[\theta_{c}-(1 / 2) \theta_{\rho} \pm(1 / 2) \theta_{-}\right]} .
\end{gathered}
$$

In each equation, the top and bottom signs are for $k_{y}=0$ and $\pi$, respectively. We omit the Klein factors for simplicity since we will not need them here. Also, we do not show combinations that can be obtained from the above by reversing all momenta.

The microscopic boson density has contributions from both the $d_{1}$ and $d_{2}$ particles via Eq. (A10), while the rung current has contributions only from the $d_{1}$ via Eq. (A11). We list all involved bilinears by their momentum:

$$
\begin{aligned}
& (0,0): \sum_{k_{y}, P} d_{1 P}^{\left(k_{y}\right) \dagger} d_{1 P}^{\left(k_{y}\right)}=\frac{\partial_{x}\left(\theta_{1}^{(0)}+\theta_{1}^{(\pi)}\right)}{\pi} ; \\
& \left(2 k_{F 1}^{\left(k_{y}\right)}, 0\right): d_{1 R}^{\left(k_{y}\right) \dagger} d_{1 L}^{\left(k_{y}\right)}=e^{-i\left(\theta_{c}+\theta_{\rho}^{ \pm} \theta_{-}\right)} ; \\
& \left(k_{F 1}^{(0)}-k_{F 1}^{(\pi)}, \pi\right): d_{1 R}^{(0) \dagger} d_{1 R}^{(\pi)}=\eta_{1}^{(0)} \eta_{1}^{(\pi)} e^{-i 2 \phi_{-}} e^{-i \theta_{-}} ; \\
& d_{1 L}^{(\pi) \dagger} d_{1 L}^{(0)}=\eta_{1}^{(\pi)} \eta_{1}^{(0)} e^{i 2 \phi_{-}} e^{-i \theta_{-}} ; \\
& \left(k_{F 1}^{(0)}+k_{F 1}^{(\pi)}, \pi\right): d_{1 R}^{(0) \dagger} d_{1 L}^{(\pi)}=\eta_{1}^{(0)} \eta_{1}^{(\pi)} e^{-i 2 \phi_{-}} e^{-i\left(\theta_{c}+\theta_{\rho}\right)} ; \\
& d_{1 R}^{(\pi) \dagger} d_{1 L}^{(0)}=\eta_{1}^{(\pi)} \eta_{1}^{(0)} e^{i 2 \phi_{-}} e^{-i\left(\theta_{c}+\theta_{\rho}\right)} ; \\
& (0,0): \sum_{P} d_{2 P}^{\dagger} d_{2 P}=\frac{\partial_{x} \theta_{2}}{\pi} ;
\end{aligned}
$$

$$
\left(2 k_{F 2}, 0\right): d_{2 R}^{\dagger} d_{2 L}=e^{i\left(\theta_{c}-2 \theta_{\rho}\right)} .
$$

Here we include the Klein factors, but we do not show combinations that can be obtained from the above by reversing all momenta.

When we include gauge fluctuations, $\theta_{c}$ becomes massive, locking together $\theta_{1}^{(0)}+\theta_{1}^{(\pi)}$ and $\theta_{2}$. Integrating out $\left(\phi_{c}, \theta_{c}\right)$, we obtain a generic harmonic liquid theory in terms of the two coupled modes $\left(\phi_{\rho}, \theta_{\rho}\right)$ and $\left(\phi_{-}, \theta_{-}\right)$. Of the cosine interactions Eq. (A4), we have only

$$
h_{4 \mathrm{ferm}}^{\mathrm{II}}=2 E_{1} \cos \left(4 \phi_{-}\right) \text {. }
$$

Assuming this is irrelevant, we have a stable phase with two gapless modes. The potential instability due to this term is considered in Appendix A 2.

We do not write explicitly the full $\mathrm{DBL}[2,1]$ theory in terms of $\left(\phi_{\rho}, \theta_{\rho}\right)$ and $\left(\phi_{-}, \theta_{-}\right)$since even without the additional interactions from Eq. (A3) we have a general coupled harmonic system. We can still make some observations about the scaling dimensions of the contributions to the boson and the boson density or current operators. In the initial fermionic mean field, the bands $d_{1}^{(0 / \pi)}, d_{2}$ are decoupled and the scaling dimensions of all contributions to the operators $b$ and $\hat{\rho}_{b}$ are equal to 1 . The gauge fluctuations effectively set $\theta_{c}$ $=0$ everywhere.

Consider first the contributions to $b$. For arbitrary couplings in the full harmonic theory, we can argue that the scaling dimensions of the operators $d_{1 R}^{\left(k_{v}\right)} d_{2 R}$ are at least 1 , i.e., larger than the mean-field value (these have no explicit Amperean enhancement). On the other hand, setting $\theta_{c}=0$, the scaling dimensions of the operators $d_{1 R}^{\left(k_{v}\right)} d_{2 L}$ can be lower than the mean field in accord with the Amperean rule. Without further information, we cannot say much-in general, these scaling dimensions can be as low as zero.

Consider now the contributions to the boson density $\hat{\rho}_{b}$. The zero-momentum contribution has scaling dimension 1 . We can argue for arbitrary couplings in the theory that the $\left(k_{F 1}^{(0)}-k_{F 1}^{(\pi)}, \pi\right)$ contribution has a scaling dimension larger than the mean-field value of 1 . On the other hand, the $\left(2 k_{F 1}^{\left(k_{y}\right)}, 0\right),\left(k_{F 1}^{(0)}+k_{F 1}^{(\pi)}, \pi\right)$, and $\left(2 k_{F 2}, 0\right)$ contributions can have smaller scaling dimensions upon setting $\theta_{c}=0$, in accord with the Amperean enhancement rule. We do not have general bounds on the scaling dimensions in the coupled two-mode system, although clearly these are not all independent. Furthermore, in all of the preceding discussion, even if the gauge interaction acts to enhance some correlation, the shortrange interactions in one dimension can act to suppress the correlation, so the ultimate fate is not clear.

As an illustration, we list all scaling dimensions in the case where the $\left(\phi_{\rho}, \theta_{\rho}\right)$ and $\left(\phi_{-}, \theta_{-}\right)$modes are decoupled and characterized by the Luttinger parameters $g_{\rho}$ and $g_{-}$, respectively [our convention is that $g$ enters as $g\left(\partial_{x} \phi\right)^{2}$ $+\frac{1}{g}\left(\partial_{x} \theta\right)^{2}$ in the action]:

$$
\begin{gathered}
\Delta\left[b_{\left(k_{F 1}^{\left(k_{y}\right)}+k_{F 2}, k_{y}\right)}\right]=\frac{3}{8}\left(\frac{2}{3 g_{\rho}}+\frac{3 g_{\rho}}{2}\right)+\frac{1}{8}\left(\frac{2}{g_{-}}+\frac{g_{-}}{2}\right) \geq 1, \\
\Delta\left[b_{\left(k_{F 1}^{\left(k_{y}\right)}-k_{F 2}, k_{y}\right)}\right]=\frac{1}{8}\left(\frac{2}{g_{\rho}}+\frac{g_{\rho}}{2}\right)+\frac{1}{8}\left(\frac{2}{g_{-}}+\frac{g_{-}}{2}\right) \geq \frac{1}{2},
\end{gathered}
$$




$$
\begin{aligned}
& \Delta\left[\rho_{b,\left(2 k_{F 1}^{\left(k_{y}\right)}, 0\right)}\right]=\frac{1}{4} g_{\rho}+\frac{1}{4} g_{-}, \\
& \Delta\left[\rho_{b,\left(k_{F 1}^{(0)}-k_{F 1}^{(\pi)}, \pi\right)}\right]=\frac{1}{2}\left(\frac{2}{g_{-}}+\frac{g_{-}}{2}\right) \geq 1, \\
& \Delta\left[\rho_{b,\left(k_{F 1}^{(0)}+k_{F 1}^{(\pi)}, \pi\right)}\right]=\frac{1}{g_{-}}+\frac{1}{4} g_{\rho}, \\
& \Delta\left[\rho_{b,\left(2 k_{F 2}, 0\right)}\right]=g_{\rho} .
\end{aligned}
$$

This example is loosely motivated by the observation in Fig. 8 that the boson $n(\mathbf{q})$ has roughly similar singularities at $\left(k_{F 1}^{(0)}-k_{F 2}, 0\right)$ and $\left(k_{F 1}^{(\pi)}-k_{F 2}, \pi\right)$. Also, increasing the ring term $K$ and decreasing the interchain hopping $J_{\perp}$ drives the mean-field bonding and antibonding bands to be more similar, suggesting such approximate decoupling of the two modes. Continuing with this illustration, the boson correlators at $\left(k_{F 1}^{\left(k_{y}\right)}-k_{F 2}, k_{y}\right)$ are most enhanced when $g_{\rho}=2$ and $g_{-}$ $=2$, giving $\Delta\left[b_{\left(k_{F 1}^{\left(k_{y}\right)}-k_{F 2}, k_{y}\right)}\right]=1 / 2$. With such couplings we also find mean-field-like $\Delta\left[\rho_{b,\left(2 k_{F 1}^{\left(k_{y}\right)}, 0\right)}\right]=\Delta\left[\rho_{b,\left(k_{F 1}^{(0)}-k_{F 1}^{(\pi)}, \pi\right)}\right]$ $=\Delta\left[\rho_{b,\left(k_{F 1}^{(0)}+k_{F 1}^{(\pi)}, \pi\right)}\right]=1$, while $\Delta\left[\rho_{b,\left(2 k_{F 2}, 0\right)}\right]=2$. These numbers are qualitatively similar to the singularities detectable by eye in the density structure factor in Fig. 8, where we see some signatures at $\left(2 k_{F 1}^{(0 / \pi)}, 0\right),\left(k_{F 1}^{(0)} \pm k_{F 1}^{(\pi)}, \pi\right)$ and no visible signature at $\left(2 k_{F 2}, 0\right)$. A more careful look at the DMRG data shows that the density singularities at $\left(2 k_{F 1}^{(0 / \pi)}, 0\right)$ are slightly stronger than the mean field, while the ones at $\left(k_{F 1}^{(0)} \pm k_{F 1}^{(\pi)}, \pi\right)$ are weaker. This would suggest that $g_{-}$is somewhat smaller than 2, which would also be consistent with the assumed irrelevance of the cosine interaction [Eq. (A15)]. However the DMRG boson correlator singularity has estimated scaling dimension somewhat smaller than the maximum $1 / 2$ in this illustration, so one should not take the above too literally. Nevertheless, this example gives some sense to the strengths of the observed singularities in Fig. 8.

Finally, consider a boson-pair operator; we list contributing four-fermion combinations by their momentum along the chains:

$$
\begin{gathered}
0: d_{1 R}^{\left(k_{y}\right)} d_{1 L}^{\left(k_{y}\right)} d_{2 R} d_{2 L} \sim e^{i\left(2 \phi_{\rho} \pm 2 \phi_{-}\right)} ; \\
P\left(k_{F 1}^{(0)}+k_{F 1}^{(\pi)}\right): d_{1 P}^{(0)} d_{1 P}^{(\pi)} d_{2 R} d_{2 L} \sim e^{i 2 \phi_{\rho}} e^{i P\left(\theta_{c}+\theta_{\rho}\right)} ; \\
P\left(k_{F 1}^{(0)}-k_{F 1}^{(\pi)}\right): d_{1 P}^{(0)} d_{1-P}^{(\pi)} d_{2 R} d_{2 L} \sim e^{i 2 \phi_{\rho}} e^{i P \theta_{-}} .
\end{gathered}
$$

As explained earlier, we characterize each contribution by the internal pair wave function [Eq. (A12)]. Again we have omitted the Klein factors but can easily restore them when needed.

For the zero-momentum contribution, we have

$$
\Phi_{1 R\left(k_{y}\right), 1 L\left(k_{y}\right), 2 R, 2 L}\left(y_{1}, y_{2}, \xi\right)=4 e^{i k_{y}\left(y_{1}+y_{2}\right)} \sin \left(k_{F 1}^{\left(k_{y}\right)} \xi\right) \sin \left(k_{F 2} \xi\right) .
$$

This remains unchanged if we simultaneously change the $y$ coordinates of both bosons in the pair, e.g., if we move a pair that lies entirely in chain 1 to chain 2 or if we turn a $+45^{\circ}$ diagonal pair into a $-45^{\circ}$ diagonal pair; we call this $s$-wave.

For the contribution at momentum $k_{F 1}^{(0)}+k_{F 1}^{(\pi)}=2 \pi \rho$, we have

$$
\begin{aligned}
& \Phi_{1 R(0), 1 R(\pi), 2 R, 2 L}\left(y_{1}, y_{2}, \xi\right)=4 \sin \left(k_{F 2} \xi\right) e^{i \pi\left(y_{1}+y_{2}\right) / 2} \sin \left[\frac{k_{F 1}^{(0)}-k_{F 1}^{(\pi)}}{2} \xi-\frac{\pi}{2}\left(y_{1}-y_{2}\right)\right] \\
& =4 \sin \left(k_{F 2} \xi\right)\left[\delta_{y_{1}=y_{2}}(-1)^{y_{1}} \sin \left(\frac{k_{F 1}^{(0)}-k_{F 1}^{(\pi)}}{2} \xi\right)+i \delta_{y_{1} \neq y_{2}}(-1)^{y_{1}} \cos \left(\frac{k_{F 1}^{(0)}-k_{F 1}^{(\pi)}}{2} \xi\right)\right] .
\end{aligned}
$$

This changes sign if we simultaneously change both $y$ coordinates in the pair. When $k_{F 1}^{(0)}$ and $k_{F 1}^{(\pi)}$ approach each other, the pairs straddling the two chains have larger amplitude. Since the amplitude changes sign when we turn $\mathrm{a}+45^{\circ}$ diagonal pair into a $-45^{\circ}$ diagonal pair, the internal structure of the pair has some $d$-wave character. The contribution at $k_{F 1}^{(0)}$ $-k_{F 1}^{(\pi)}$ can be similarly characterized but is omitted here.

In the free fermion mean field, all contributions have scaling dimension 2 . Beyond the mean field, the $d$-wave contribution at $k_{F 1}^{(0)}+k_{F 1}^{(\pi)}$ is potentially enhanced by setting $\theta_{c}=0$. Still, its scaling dimension is at least 1 , while we cannot say much about the scaling dimension of the $s$-wave contribution at zero momentum. In the above illustration with decoupled $\phi_{\rho}$ and $\phi_{-}$modes, the $d$-wave pair has scaling dimension $1 / g_{\rho}+g_{\rho} / 4=1$ if we set $g_{\rho}=2$, while the $s$-wave pair has a scaling dimension of $1 / g_{\rho}+1 / g_{-}=1$ if we also set $g_{-}=2$. The latter would be larger if we take smaller $g_{-}$, in agreement with the observed dominance of the $d$-wave-like pair correlations in the DMRG (Fig. 10) oscillating at wave vector $2 \pi \rho$.

\section{Possible instability of DBL[2,1]}

Let us ask what phase is obtained starting from DBL[2,1] if the interaction in Eq. (A15) is relevant [the Amperean rule does not point one way or another, but additional interactions from Eq. (A3) can make this happen]. Then $\phi_{-}$is pinned, with two distinct cases depending on the sign of $E_{1}$ considered below, while $\theta_{-}$fluctuates wildly. All contributions to $b$ contain $\theta_{-}$in the exponent, so the single-boson correlation 
function decays exponentially-there is a charge gap to single-boson excitations. The $\left(\phi_{\rho}, \theta_{\rho}\right)$ mode still remains gapless, and we have a single-mode harmonic fluid described by the parameter $g_{\rho}$.

The boson density shows power-law correlations at zero momentum with scaling dimension 1. The remaining particle-hole bilinears with power-law correlations are at wave vector $\left(k_{F 1}^{(0)}+k_{F 1}^{(\pi)}, \pi\right)=(2 \pi \rho, \pi)$ (scaling dimension: $\left.g_{\rho} / 4\right)$ and at $\left(2 k_{F 2}, 0\right)=(4 \pi \rho, 0)$ (scaling dimension: $\left.g_{\rho}\right)$. The $\mathbf{q}=0$ and $\mathbf{q}=\left(2 k_{F 2}, 0\right)$ parts do not distinguish qualitatively between the two possibilities $E_{1}>0$ and $E_{1}<0$, while the $\mathbf{q}=\left(k_{F 1}^{(0)}+k_{F 1}^{(\pi)}, \pi\right)$ part does distinguish. Explicitly, the $d_{1}$-particle density is

$$
\rho_{d_{1}}(x, y)=-2 i \eta_{1}^{(0)} \eta_{1}^{(\pi)} \sin \left(2 \phi_{-}\right) e^{i \theta_{\rho}} e^{i\left(k_{F 1}^{(0)}+k_{F 1}^{(\pi)}\right) x+i \pi y}+\text { H . c . , }
$$

where we have omitted the zero-momentum component and parts with exponentially vanishing correlations. On the other hand, the $d_{1}$-particle rung current is

$$
j_{\perp, 1}(x)=2 i \eta_{1}^{(0)} \eta_{1}^{(\pi)} \cos \left(2 \phi_{-}\right) e^{i \theta_{\rho}} e^{i\left(k_{F 1}^{(0)}+k_{F 1}^{(\pi)}\right) x}+\text { H . c . }
$$

Consider now two cases:

(a) $E_{1}>0$ : It follows that $2 \phi_{-}=\frac{\pi}{2} \bmod \pi$. In this case $\rho_{d_{1}}$ shows power-law correlations at $\left(k_{F 1}^{(0)}+k_{F 1}^{(\pi)}, \pi\right)$, while $j_{\perp, 1}$ correlations are absent.

(b) $E_{1}<0$ : It follows that $2 \phi_{-}=0 \bmod \pi$. In this case $\rho_{d_{1}}$ correlations at the above wave vector are absent, while $j_{\perp, 1}$ shows power law at wave vector $k_{F 1}^{(0)}+k_{F 1}^{(\pi)}$ along the chains.

Let us finally consider the pair-boson correlations, which are also power law. Putting together all contributions to $b\left(x_{1}, y_{1}\right) b\left(x_{2}, y_{2}\right)$, we have

$$
\begin{aligned}
& e^{i 2 \phi_{\rho}}\left[e^{i 2 \phi_{-}} \Phi_{1 R(0), 1 L(0), 2 R, 2 L}+e^{-i 2 \phi_{-}} \Phi_{1 R(\pi), 1 L(\pi), 2 R, 2 L}\right] \\
& +\sum_{P} \eta_{1}^{(0)} \eta_{1}^{(\pi)} e^{i\left\{2 \phi_{\rho}+P\left[\theta_{\rho}+\left(k_{F 1}^{(0)}+k_{F 1}^{(\pi)}\right) X\right]\right\}} \Phi_{1 P(0), 1 P(\pi), 2 R, 2 L},
\end{aligned}
$$

where the appropriate $\Phi\left(y_{1}, y_{2}, \xi\right)$ are given in Eqs. (A16) and (A17). The pairs at zero momentum have dominant correlations with scaling dimension $1 / g_{\rho}$. These have $s$-wave character as far as rotating diagonal bonds is concerned, but the amplitude details depend on the sign of $E_{1}$. The pairs at $k_{F 1}^{(0)}+k_{F 1}^{(\pi)}$ have subdominant correlations with scaling dimension $1 / g_{\rho}+g_{\rho} / 4$. These have $d$-wave character for rotating the diagonal bonds and the details do not depend much on the sign of $E_{1}$.

Summarizing, the resulting phase has a gap to singleboson excitations but has power-law correlations for particlehole and particle-particle composites. The dominant particlehole composite has scaling dimension $g_{\rho} / 4$ and contributes either to the density at wave vector $(2 \pi \rho, \pi)$ if $E_{1}>0$ or to the rung current at $2 \pi \rho$ if $E_{1}<0$. The dominant particleparticle composite has scaling dimension $1 / g_{\rho}$ and represents $s$-wave pairing at zero momentum. Loosely speaking, we can describe this phase by saying that the bosons form $s$-wave-like pairs and these molecules in turn form a Luttinger liquid. The current and/or density fluctuations occur at a wavelength equal to the mean interpair spacing along the ladder, as expected for a 1D Luttinger liquid (of pairs). The form of the particle-hole fluctuations across the rungs pre- sumably reflects the internal pair structure in the two cases.

\section{DBL[2,2] and instability toward $s$-wave pairing}

In the DBL[2,2] case, we start with four bands, $d_{1}^{(0 / \pi)}, d_{2}^{(0 / \pi)}$, and have four modes upon bosonization [Eq. (A7)]. It is convenient to introduce the following canonical variables:

$$
\begin{aligned}
& \phi_{\rho}=\frac{1}{2}\left(\phi_{1}^{(0)}+\phi_{1}^{(\pi)}+\phi_{2}^{(0)}+\phi_{2}^{(\pi)}\right), \\
& \phi_{c}=\frac{1}{2}\left(\phi_{1}^{(0)}+\phi_{1}^{(\pi)}-\phi_{2}^{(0)}-\phi_{2}^{(\pi)}\right), \\
& \phi_{+}=\frac{1}{2}\left(\phi_{1}^{(0)}-\phi_{1}^{(\pi)}+\phi_{2}^{(0)}-\phi_{2}^{(\pi)}\right), \\
& \phi_{-}=\frac{1}{2}\left(\phi_{1}^{(0)}-\phi_{1}^{(\pi)}-\phi_{2}^{(0)}+\phi_{2}^{(\pi)}\right),
\end{aligned}
$$

with the same transformation for the $\theta$ variables. The cosine interactions $[\mathrm{Eq} .(\mathrm{A} 4)]$ are

$$
\begin{aligned}
h_{4 \mathrm{ferm}}^{(\mathrm{II})}= & 2 E_{1} \cos \left(2 \phi_{+}+2 \phi_{-}\right)+2 E_{2} \cos \left(2 \phi_{+}-2 \phi_{-}\right) \\
& -4 \hat{\Gamma} F \cos \left(2 \phi_{+}\right) \cos \left(2 \theta_{c}\right)+4 \hat{\Gamma} G \cos \left(2 \phi_{-}\right) \cos \left(2 \theta_{c}\right),
\end{aligned}
$$

where $\hat{\Gamma}=\eta_{1}^{(0)} \eta_{1}^{(\pi)} \eta_{2}^{(0)} \eta_{2}^{(\pi)}$.

Proceeding with the analysis as in the DBL[2,1] case, upon integrating out the gauge field, $\theta_{c}$ becomes massive. If by tweaking the strictly marginal interactions $h_{4 \text { ferm }}^{(\mathrm{I})}$ we could render the $h_{4 \text { ferm }}^{\text {(II) }}$ terms irrelevant, we would end up with a phase with three gapless modes. We call this possible phase DBL[2,2], and it can be analyzed similarly to DBL[2,1]. For example, the mean-field boson correlation in this phase would read

$$
\begin{aligned}
G_{b}^{\mathrm{MF}}(x, y) \sim & \sum_{k_{y 1}, k_{y 2}} \frac{e^{i\left(k_{y 1}+k_{y 2}\right) y}}{x^{2}}\left\{\cos \left[\left(k_{F 1}^{\left(k_{y 1}\right)}-k_{F 2}^{\left(k_{y 2}\right)}\right) x\right]\right. \\
& \left.-\cos \left[\left(k_{F 1}^{\left(k_{y 1}\right)}+k_{F 2}^{\left(k_{y 2}\right)}\right) x\right]\right\} .
\end{aligned}
$$

The first term in the brackets comes from $d_{1 P}^{\left(k_{11}\right)} d_{2-P}^{\left(k_{y 2}\right)}$ and is expected to be enhanced by Amperean attraction. (In the bosonization, this term contains $\theta_{c}$ in the exponent and is potentially enhanced upon setting $\theta_{c}=0$.) On the other hand, the second term in the brackets has no Amperean enhancement. As emphasized earlier, besides the gauge fluctuations crudely captured by the Amperean rule, other interactions can also change the scaling dimensions. Interestingly, and this has important consequences explored below, $d_{1 R}^{(0)} d_{2 L}^{(0)}$ and $d_{1 L}^{(\pi)} d_{2 R}^{(\pi)}$ carry the same momentum $\left(k_{F 1}^{(0)}-k_{F 2}^{(0)}, 0\right)=\left(k_{F 2}^{(\pi)}\right.$ $\left.-k_{F 1}^{(\pi)}, 0\right)$, and also $d_{1 R}^{(0)} d_{2 L}^{(\pi)}$ and $d_{1 L}^{(\pi)} d_{2 R}^{(0)}$ carry the same momentum $\left(k_{F 1}^{(0)}-k_{F 2}^{(\pi)}, \pi\right)=\left(k_{F 2}^{(0)}-k_{F 1}^{(\pi)}, \pi\right)$. In fact, Josephsontype coupling between the first two "bosonic modes" is allowed and gives rise to the $F$ interaction in Eq. (A4), while Josephson coupling between the last two modes gives rise to the $G$ interaction. If the gauge physics is the dominant determining factor in the DBL[2,2] phase, we expect the state to 
be strongly unstable to these interactions, and we pursue this scenario below. On the other hand, if DBL[2,2] were stabilized against $F$ and $G$ by some density-density interactions dominating over the gauge physics, which is possible and interesting, we would have little intuition from the gauge theory perspective, and we do not pursue this possibility further.

If the gauge physics dominates, it appears very likely that the $F$ and $G$ terms are relevant, as can be seen from their bosonized expressions upon setting $\theta_{c}=0$ (in accord with the Amperean rule). Let us explore the resulting phase when $F$ and $G$ flow to large values and pin the fields $\phi_{+}$and $\phi_{-}$. Four different possibilities depending on the signs of $F$ and $G$ are discussed below. In such a phase, the conjugate fields $\theta_{+}$and $\theta_{-}$fluctuate strongly and any operator containing these in the exponent will have only short-range correlations. Thus, we conclude that the boson correlations decay exponentially; i.e., there is a gap to single-boson excitations. However, there is still one gapless mode $\left(\phi_{\rho}, \theta_{\rho}\right)$, which we characterize with the Luttinger parameter $g_{\rho}$.

Consider fermion bilinears composed of a particle and a hole of the same species that enter density and current [Eqs. (A10) and (A11)]. Other than the $q=0$ densities $d_{\alpha P}^{\left(k_{y}\right) \dagger} d_{\alpha P}^{\left(k_{y}\right)}$, the following bilinears survive when $\phi_{ \pm}$get pinned:

$$
\begin{aligned}
& d_{\alpha P}^{(0) \dagger} d_{\alpha-P}^{(\pi)}=\eta_{\alpha}^{(0)} \eta_{\alpha}^{(\pi)} e^{-i\left(\phi_{+}+e_{\alpha} \phi_{-}\right)} e^{-i P\left(\theta_{\rho}+e_{\alpha} \theta_{c}\right)}, \\
& d_{\alpha P}^{(\pi) \dagger} d_{\alpha-P}^{(0)}=\eta_{\alpha}^{(\pi)} \eta_{\alpha}^{(0)} e^{i\left(\phi_{+}+e_{\alpha} \phi_{-}\right)} e^{-i P\left(\theta_{\rho}+e_{\alpha} \theta_{c}\right)} .
\end{aligned}
$$

The particle density [Eq. (A10)] is, upon dropping the zeromomentum part and setting $\theta_{c}=0$,

$$
\begin{aligned}
\rho_{d_{\alpha}}(x, y)= & -2 i \eta_{\alpha}^{(0)} \eta_{\alpha}^{(\pi)} \sin \left(\phi_{+}\right. \\
& \left.+e_{\alpha} \phi_{-}\right) e^{i \pi y} \sum_{P} e^{i P \theta_{\rho}} e^{i P\left(k_{F \alpha}^{(0)}+k_{F \alpha}^{(\pi)}\right) x} .
\end{aligned}
$$

The particle current on the rungs $[\mathrm{Eq} .(\mathrm{A} 11)]$ is

$$
j_{\perp, \alpha}(x)=2 i \eta_{\alpha}^{(0)} \eta_{\alpha}^{(\pi)} \cos \left(\phi_{+}+e_{\alpha} \phi_{-}\right) \sum_{P} e^{i P \theta_{\rho}} e^{i P\left(k_{F \alpha}^{(0)}+k_{F \alpha}^{(\pi)}\right) x} .
$$

We now discuss different phases that can arise depending on the signs of the couplings $F$ and $G$. We set $\Gamma=1$, from which it follows that $\left\langle\eta_{2}^{(0)} \eta_{2}^{(\pi)}\right\rangle=-\left\langle\eta_{1}^{(0)} \eta_{1}^{(\pi)}\right\rangle$ (the physical results are independent of the choice of $\Gamma$ ). There are four cases:

(a) $F>0, G>0$ : It follows that $\phi_{+}=0 \bmod \pi, \phi_{-}$ $=\frac{\pi}{2} \bmod \pi$. In this case $\rho_{d_{1}}=\rho_{d_{2}}$ show power-law correlations at $(2 \pi \rho, \pi)$ with the scaling dimension $g_{\rho} / 4$, while $j_{\perp, \alpha}$ correlations are absent. This is a natural phase coming from the microscopic gauge theory since the fluctuations of the $d_{1}$ and $d_{2}$ densities are in sync. Also, when we integrate out the massive gauge field $a_{\mathrm{I}}-a_{\mathrm{II}}$ early in the derivation of the continuum theory, we in fact generate such $F=G>0$ couplings. Foretelling the analysis of the pairing correlations below, we propose that this is the $s$-wave-paired phase observed in the DMRG (Sec. III C).

(b) $F<0, G<0$ : It follows that $\phi_{+}=\frac{\pi}{2} \bmod \pi, \phi_{-}$ $=0 \bmod \pi$. In this case $\rho_{d_{1}}=-\rho_{d_{2}}$ show power-law correla- tions at $(2 \pi \rho, \pi)$, while $j_{\perp, \alpha}$ correlations are absent. The fluctuations of the $d_{1}$ and $d_{2}$ densities are out of sync. This phase with spatial modulation of the gauge charge is less natural coming from the lattice theory. It is in principle allowed if the bare couplings are finite, since then such modulation costs only finite-energy density that can be offset by some other short-range interactions. However the cost is large if the microscopic theory is at strong coupling as is usually the case in the slave-particle treatments. For example, only the phase with $\rho_{d_{1}}=\rho_{d_{2}}$ can be realized by our wave functions.

(c) $F<0, G>0$ : It follows that $\phi_{+}=\phi_{-}=\frac{\pi}{2} \bmod \pi$. In this case $\rho_{d_{\alpha}}$ correlations are absent, while $j_{\perp, 1}=j_{\perp, 2}$ show power-law correlations at wave vector $2 \pi \rho$ along the chains with scaling dimension $g_{\rho} / 4$. The $d_{1^{-}}$and $d_{2^{-}}$particle currents are in sync, so this phase is natural in the gauge theory. For the original bosons, it would have enhanced currentcurrent correlations but not density correlations. However, we have not observed such a phase in the ring model.

(d) $F>0, G<0$ : It follows that $\phi_{+}=\phi_{-}=0 \mathrm{mod} \pi$. In this case $\rho_{d_{\alpha}}$ correlations are absent, while $j_{\perp, 1}=-j_{\perp, 2}$ show power-law correlations at wave vector $2 \pi \rho$ along the chains. Since the currents are out of sync, this phase is less natural coming from the microscopic gauge theory.

Let us consider boson pairs. First, for each $\alpha=1,2$ there are four $d_{\alpha} d_{\alpha}$ continuum field combinations that survive when the $\phi_{+}$are pinned:

$$
\begin{gathered}
d_{\alpha P}^{(0)} d_{\alpha P}^{(\pi)}=\eta_{\alpha}^{(0)} \eta_{\alpha}^{(\pi)} e^{i\left(\phi_{\rho}+e_{\alpha} \phi_{c}\right)} e^{i P\left(\theta_{\rho}+e_{\alpha} \theta_{c}\right)}, \\
d_{\alpha R}^{\left(k_{y}\right)} d_{\alpha L}^{\left(k_{y}\right)}=e^{i\left[\phi_{\rho}+e_{\alpha} \phi_{c} \pm\left(\phi_{+}+e_{\alpha} \phi_{-}\right)\right]},
\end{gathered}
$$

where in the last line the top/bottom sign corresponds to $k_{y}$ $=0 / \pi$. Constructing boson pairs via $d_{1} d_{1} d_{2} d_{2}$, the field $\phi_{c}$ disappears, and we also set $\theta_{c}=0$. Of the 16 combinations, 6 carry zero momentum and all have the largest scaling dimension $1 / g_{\rho}$ :

$$
\begin{gathered}
d_{1 P}^{(0)} d_{1 P}^{(\pi)} d_{2-P}^{(0)} d_{2-P}^{(\pi)}=\eta_{1}^{(0)} \eta_{1}^{(\pi)} \eta_{2}^{(0)} \eta_{2}^{(\pi)} e^{i 2 \phi_{\rho},} \\
d_{1 R}^{\left(k_{y}\right)} d_{1 L}^{\left(k_{y}\right)} d_{2 R}^{\left(k_{y}\right)} d_{2 L}^{\left(k_{y}\right)}=e^{i\left(2 \phi_{\rho} \pm 2 \phi_{+}\right)}, \\
d_{1 R}^{\left(k_{y}\right)} d_{1 L}^{\left(k_{y}\right)} d_{2 R}^{\left(k_{y}+\pi\right)} d_{2 L}^{\left(k_{y}+\pi\right)}=e^{i\left(2 \phi_{\rho} \pm 2 \phi_{-}\right)} .
\end{gathered}
$$

The second and third lines will likely have smaller amplitudes, since $\phi_{+}$and $\phi_{-}$still fluctuate a little about the pinned values.

We now examine the internal pairing structures. Corresponding to the first line above, we have:

$$
\begin{aligned}
\Phi_{1 P(0), 1 P(\pi), 2-P(0), 2-P(\pi)}\left(y_{1}, y_{2}, \xi\right) \\
=-4 \delta_{y_{1}=y_{2}} \sin \left(\frac{k_{F 1}^{(0)}-k_{F 1}^{(\pi)}}{2} \xi\right) \sin \left(\frac{k_{F 2}^{(0)}-k_{F 2}^{(\pi)}}{2} \xi\right) \\
-4 \delta_{y_{1} \neq y_{2}} \cos \left(\frac{k_{F 1}^{(0)}-k_{F 1}^{(\pi)}}{2} \xi\right) \cos \left(\frac{k_{F 2}^{(0)}-k_{F 2}^{(\pi)}}{2} \xi\right) .
\end{aligned}
$$

The $\delta_{y_{1}=y_{2}}$ piece describes a pair formed by bosons in the same chain, while $\delta_{y_{1} \neq y_{2}}$ is a pair straddling the two chains. 
When, say, $k_{F 1}^{(0)} \approx k_{F 1}^{(\pi)}$, which becomes more accurate as we decrease $J_{\perp}$, the amplitudes for the latter are significantly stronger; i.e., pairs are predominantly straddling the two chains.

Corresponding to the other zero-momentum combinations, we have

$$
\begin{aligned}
& \Phi_{1 R\left(k_{y}\right), 1 L\left(k_{y}\right), 2 R\left(k_{y}^{\prime}\right), 2 L\left(k_{y}^{\prime}\right)}\left(y_{1}, y_{2}, \xi\right) \\
& =4 e^{i\left(k_{y}+k_{y}^{\prime}\right)\left(y_{1}+y_{2}\right)} \sin \left(k_{F 1}^{\left(k_{y}\right)} \xi\right) \sin \left(k_{F 2}^{\left(k_{y}^{\prime}\right)} \xi\right) .
\end{aligned}
$$

If $k_{y}=k_{y}^{\prime}$, this is independent of whether the two sites are on the same or different chains. On the other hand, if $k_{y} \neq k_{y}^{\prime}$, this has opposite signs if the two sites are on the same instead of different chains.

All of the above pair functions are even if both particles are moved perpendicular to the chains. In particular, $+45^{\circ}$ and $-45^{\circ}$ diagonal pairs have the same amplitudes. This is what we call $s$-wave pairing. Putting together all zeromomentum contributions to $b\left(x_{1}, y_{1}\right) b\left(x_{2}, y_{2}\right)$, we have

$$
\begin{aligned}
& e^{i 2 \phi_{\rho}\left[2 \Gamma \Phi_{1 R(0), 1 R(\pi), 2 L(0), 2 L(\pi)}\right.} \\
& +e^{i 2 \phi_{+}} \Phi_{1 R(0), 1 L(0), 2 R(0), 2 L(0)} \\
& +e^{-i 2 \phi_{+}} \Phi_{1 R(\pi), 1 L(\pi), 2 R(\pi), 2 L(\pi)} \\
& +e^{i 2 \phi_{-}} \Phi_{1 R(0), 1 L(0), 2 R(\pi), 2 L(\pi)} \\
& \left.+e^{-i 2 \phi_{-}} \Phi_{1 R(\pi), 1 L(\pi), 2 R(0), 2 L(0)}\right]
\end{aligned}
$$

where $\Gamma=\eta_{1}^{(0)} \eta_{1}^{(\pi)} \eta_{2}^{(0)} \eta_{2}^{(\pi)}$ and eventually drops out since both $\Gamma \cos \left(2 \phi_{+}\right)$and $\Gamma \cos \left(2 \phi_{-}\right)$are determined uniquely by the signs of the $F$ and $G$ couplings: $\Gamma e^{ \pm i 2 \phi_{+}}=\operatorname{sgn}(F)$, $\Gamma e^{ \pm i 2 \phi_{-}}=-\operatorname{sgn}(G)$. Note also that the contributions in the last four lines will have smaller amplitudes since $\phi_{+}$and $\phi_{-}$fluctuate a little around the pinned values. So the numerically largest contribution is given by the first line, while the smaller contributions from the last four lines will add to it with relative signs that depend on the signs of the $F$ and $G$.

Summarizing, DBL[2,2] is unstable toward a bosonpaired phase with $s$-wave pairs carrying zero momentum. The resulting phase is roughly similar to the $s$-wave-paired phase discussed as a possible instability of DBL[2,1] in Appendix A 2, but some details are different. For example, coming out of DBL[2,1], the amplitude for rung pairs vanishes, while out of the $\mathrm{DBL}[2,2]$ the rung pairs have large amplitudes comparable to those of the diagonal pairs. The latter is more similar to what the DMRG finds in the $s$-wave-paired phase in the ring model (Sec. III C). The prediction of dominant density correlations at wave vector $(2 \pi \rho, \pi)$ also agrees with the DMRG. One additional prediction from the theory is that the pairing and density power laws have exponents that are inverse of each other. While the DMRG estimates for the present system sizes do not satisfy this exactly, there are likely strong finite-size effects, and we would like to revisit this with larger systems.

\section{APPENDIX B: LIMITING CASES IN THE $J$-K MODEL}

\section{DBL[2,1] as Jordan-Wigner in a model with no-double occupancy of rungs}

In the DBL construction [Eq. (2)], we can view one determinant as affecting a generalized flux attachment or Jordan-Wigner (JW) transformation and view the other determinant as a ground state of the new JW fermions. In the $\operatorname{DBL}[2,1]$ case, the $d_{2}$ determinant is composed entirely from the bonding orbitals and becomes Eq. (18). In particular, it prevents two bosons from being on the same rung. If we consider a hard-core boson model that prohibits double occupancy of rungs, then $\operatorname{sgn}\left(\operatorname{det}_{2}\right)$ is the conventional 1D chain JW transformation on this ladder. As can be seen from the bottom panel of Fig. 7, our ring model in the DBL[2,1] phase appears, by its own dynamics, to strongly suppress double rung occupancy. The optimized power of $\operatorname{det}_{2}$ in the trial wave function is also relatively small, making the $\operatorname{sgn}\left(\operatorname{det}_{2}\right)\left|\operatorname{det}_{2}\right|^{p}$ factor look more like Jordan-Wigner. Finally, the $d_{1}$ determinant is composed of the bonding and antibonding orbitals, and its optimized power is relatively large though smaller than 1, which suggests that the JW fermions are not far from being free despite the large ring exchanges and the restricted rung occupancy.

On the other hand, DBL[2,2] would have some double occupancy of rungs and would be an example of a nontrivial JW. However unfortunately this phase appears to be unstable as described in Appendix A 3. The instability happens because of special kinematic conditions satisfied here, which allow direct Josephson coupling and locking between enhanced boson modes. However, we expect that on $N$-leg ladders with $N \geq 3$, DBL phases will exist which cannot be described by a conventional Jordan-Wigner approach.

\section{Solution of the $K$-only model}

The pure ring model on the two-leg ladder can be solved exactly. In the absence of boson hopping terms $(J=0)$, the number of bosons on each rung, $N_{\text {rung }}(x)=n(x, 1)+n(x, 2)$, is separately conserved. The number of bosons in each chain, $N_{\text {ch.I }}$ or $N_{\text {ch.II }}$, is also conserved. Any rung with $N_{\text {rung }}=0$ or 2 effectively breaks the system into decoupled pieces.

Consider an isolated segment of $L$ rungs with each $N_{\text {rung }}$ $=1$. The ring Hamiltonian is mapped to an $X Y$ spin chain by identifying boson configuration $\{n(x, 1), n(x, 2)\}=\{1,0\}$ with spin up and $\{0,1\}$ with spin down:

$$
H[L]=K \sum_{x=1}^{L-1}\left[\tau^{+}(x) \tau^{-}(x+1)+\text { H . c. }\right],
$$

where $\tau$ are the usual spin-1/2 operators. This is readily solved by free fermions, and the ground-state energy is

$$
E_{\mathrm{gs}}[L]=-2 K \sum_{n=1}^{n_{\max }} \cos \frac{\pi n}{L+1}
$$




$$
=-K\left[\frac{\sin \frac{\pi\left(2 n_{\max }+1\right)}{2(L+1)}}{\sin \frac{\pi}{2(L+1)}}-1\right],
$$

where $n_{\max }=L / 2$ if $L$ is even (in this case $N_{\text {ch.I }}=N_{\text {ch.II }}=L / 2$ ), while $n_{\max }=(L+1) / 2$ if $L$ is odd $\left(N_{\text {ch.I }}=N_{\text {ch.II }} \pm 1\right)$. In either case, the ground-state energy per boson is minimized by making $L$ large, with the asymptotic behavior

$$
\epsilon_{\text {per boson }}=\frac{E_{\mathrm{gS}}[L]}{L}=-\frac{2 K}{\pi}+\frac{K(1-2 / \pi)}{L}+O\left(\frac{1}{L^{2}}\right) .
$$

Going back to the $K$-only model on an infinitely long ladder, we conclude that for arbitrary boson density $\rho$ $<1 / 2$, it is advantageous to phase separate into an empty region and a half-filled region, since this minimizes the energy per particle $(\rho>1 / 2$ can be treated by particle-hole transformation). The phase separation arises because the ring exchanges provide an effective attraction between particles-see also Appendix B 3. Note that the half-filled region itself is a highly correlated state of bosons.

\section{Bound state of two bosons for large $K$ and $d$-wave-paired state at low densities}

Consider two bosons on an otherwise empty ladder. In the absence of the hoppings, $J=J_{\perp}=0$, the states

$$
\frac{b(x, 1)^{\dagger} b(x+1,2)^{\dagger}-b(x, 2)^{\dagger} b(x+1,1)^{\dagger}}{\sqrt{2}}\left|\operatorname{vacuum}_{b}\right\rangle
$$

are degenerate ground states with energy $-K$. Each can be viewed as a $d$-wave pair sitting on a plaquette. Small boson hopping can be included perturbatively, and the pair starts to hop along the ladder with amplitude $J^{2} / K$. In fact, inspired by similar considerations in the $1 \mathrm{D} t-J$ model in Ref. 27 , the zero-momentum bound state can be written exactly for the general $J-J_{\perp}-K$ model and is created by

$$
\begin{gathered}
\sum_{x} \sum_{n=1}^{\infty}\left(\frac{2 J}{K}\right)^{n-1}\left[b(x, 1)^{\dagger} b(x+n, 2)^{\dagger}\right. \\
\left.-b(x, 2)^{\dagger} b(x+n, 1)^{\dagger}\right] .
\end{gathered}
$$

This is defined for $K>2 J$ and has energy

$$
E_{\text {bound }}=-K-\frac{4 J^{2}}{K} \text {. }
$$

The pair size is $\xi=1 / \log [K /(2 J)]$.

Note that the bound state does not utilize any rung hopping. Also, it competes with a free state of the two bosons where each moves independently and where the total energy is

$$
E_{\text {free }}=-2\left(2 J+J_{\perp}\right),
$$

assuming $J, J_{\perp} \geq 0$ throughout. As an example, for $J_{\perp}=0$ the bound state has lower energy than the state with two free bosons when $K>K_{c}\left[J_{\perp}=0\right]=2 J$, while $K_{c}\left[J_{\perp}=0.1 J\right]=2.74 J$ and $K_{c}$ increases with increasing $J_{\perp}$. For a very small density of bosons, it is then natural to propose that for $K>K_{c}$ the bosons will form such tightly bound pairs, which in turn form a Luttinger liquid. This is the picture of the $d$-wave-paired phase.

As discussed in Appendix B 2, in the absence of the hopping (or for very large $K$ ), the system at any density phase separates into empty and half-filled regions. This maximizes the ring energy per particle, which attains $-2 K / \pi$, and in particular wins over the asymptotic $-K / 2$ energy per particle in the paired state. However, bosons in the phase-separated half-filled region form a nontrivial quantum state and can hardly gain any kinetic energy once the hopping is included. In the boson-paired liquid, on the other hand, the pairs can move and gain some kinetic energy, which can then stabilize the system against phase separation. As a crude estimate, we ignore the kinetic energy in the phase-separated case and compare $-2 K / \pi$ with Eq. (B8) divided per boson and find that the boson-paired liquid is more stable for $K<3.8 \mathrm{~J}$. Thus, there is a sizable window over which the boson-paired liquid is energetically preferable over the free boson liquid and is stable against phase separation.

At very low density we also need to consider more-bosonbound states that successively improve their ring energy but lose kinetic energy; the corresponding liquids could intervene between the boson-paired one and the phase separation. However, it seems likely that a sizable region of the bosonpaired phase will remain. Of course, it also competes with other phases such as DBL, as happens in Fig. 4 for the filling $\rho=1 / 9$ studied by DMRG.

\section{APPENDIX C: TRIAL WAVE FUNCTIONS FOR BOSON-PAIRED PHASES}

Let us sketch how one can construct trial wave functions for boson-paired liquids such as the $d$-wave-paired phase at low densities described in Appendix B 3 and in Sec. III D and the $s$-wave-paired phase described in Sec. III C. Let us denote the boson-pair wave function as $\phi\left(r-r^{\prime}\right)$. For a given pairing up of particles, we could write $\phi\left(r_{1}-r_{2}\right) \phi\left(r_{3}-r_{4}\right) \cdots$ times some Jastrow-type factor for the center-of-mass coordinates $R_{12}=\left(r_{1}+r_{2}\right) / 2, R_{34}=\left(r_{3}+r_{4}\right) / 2, \ldots$, and we then symmetrize this to obtain a bosonic wave function:

$$
\begin{aligned}
\Psi= & \mathcal{S}\left[\phi\left(r_{1}-r_{2}\right) \phi\left(r_{3}-r_{4}\right) \cdots\right. \\
& \left.\times \operatorname{Jastrow}\left(R_{12}, R_{34}, \ldots\right)\right] .
\end{aligned}
$$

Even if we take a simpler Jastrow which is already a symmetric function of $\left\{r_{i}\right\}$, the symmetrization of the pairing part gives a Permanent of a matrix which is prohibitive in numerical calculations.

An alternative way to construct a symmetric bosonic wave function is

$$
\begin{gathered}
\Psi=\operatorname{Pf}\left[g\left(r_{1}-r_{2}\right) g\left(r_{3}-r_{4}\right) \cdots\right] \\
\times \operatorname{det}\left[r_{1}, r_{2}, r_{3}, \ldots\right] \times \operatorname{Jastrow}\left[r_{1}, r_{2}, r_{3}, \ldots\right] .
\end{gathered}
$$

Here pairing is realized with the help of a Pfaffian, which is a BCS wave function for spinless fermions and is specified 
by some pair function $g\left(r-r^{\prime}\right)$, while the total bosonic symmetry is recovered by multiplying by a second fermionic wave function taken for simplicity to be a Slater determinant of some orbitals. Note that for tightly bound pairs when $\phi\left(r-r^{\prime}\right)$ and $g\left(r-r^{\prime}\right)$ are very short ranged, the two constructions are essentially similar for statistically significant configurations. The Pfaffian and determinant are simple to work with in variational Monte Carlo (VMC), which allows one to construct trial wave functions also beyond the tightly bound limit. Of course, both the spinless fermion pair function $g\left(r-r^{\prime}\right)$ in the Pfaffian and the orbitals in the determinant need to be judiciously chosen to realize the particular boson-pair function $\phi\left(r-r^{\prime}\right)$. We should also note that such $\operatorname{det} \times \mathrm{Pf}$ wave function does not necessarily represent a paired state of bosons, and one needs to measure the longdistance properties first. For example, we found that for a 1D chain a product of a Slater determinant and a $p_{x}$-wave BCS wave function has QLRO in the boson correlator when the $p_{x}$-pairing problem is at weak coupling, while the boson correlator becomes short range-as needed for the boson-paired state-only when the $p_{x}$-pairing problem is at strong coupling.

\section{1. $d$-wave-paired wave function}

Consider for illustration low-density bosons in the $J_{\perp}=0$ limit. We can construct a $d$-wave-paired state by taking

$$
g\left(x_{1}, y_{1} ; x_{2}, y_{2}\right)=\delta_{y_{1} \neq y_{2}} \operatorname{sgn}\left(y_{1}-y_{2}\right) e^{-\left|x_{1}-x_{2}\right| / \xi}
$$

in the Pfaffian part, while combining the determinant and Jastrow into one factor,

$$
\text { det } \times \text { Jastrow }=\left[\prod_{i<j} \sin \frac{\pi\left(x_{i}-x_{j}\right)}{L}\right]^{p} .
$$

The power of the determinant is understood as (det) ${ }^{p}$ $=\operatorname{sgn}(\operatorname{det})|\operatorname{det}|^{p}$. The fermion pair function is $p_{y}$ type and here requires that the two particles be on different chains (which is why it is strictly appropriate only in the $J_{\perp}=0$ limit). For an isolated pair of bosons, this indeed gives the exact $d$-wave pair of Appendix B 3 when $p \rightarrow 0$. We use this wave function with two variational parameters $\xi$ and $p$ to connect with the DMRG results at $\rho=1 / 9$ in Sec. III D. One could construct a similar boson-paired state by taking the Pfaffian factor to be a solution of a general spinless BCS problem with $p_{y}$ pairing on the ladder. This would allow more freedom (e.g., if we want to study $J_{\perp} \neq 0$ ), but it has not been explored.

\section{2. $s$-wave-paired wave function}

We have also tried the $\operatorname{det} \times \mathrm{Pf}$ construction for the $s$-wave-paired phase in Sec. III C. Here we take the determinant to contain both bonding and antibonding orbitals, which resembles the $\operatorname{det}_{1}$ in $\mathrm{DBL}[2,1]$ or $\mathrm{DBL}[2,2]$ containing $d_{1}$ fermions with dominant hopping along the chains (in particular, the $d_{1}$ bonding and antibonding bands become equally populated in the $J_{\perp}=0$ limit). We take the Pfaffian to be that for a $p_{x}$-wave spinless BCS problem on the ladder, which resembles $d_{2}$ fermions trying to enter the DBL[2,2] regime but becoming paired. There are many parameters here such as the $d_{2}$ intra- and interchain hoppings and $p_{x}$ pairings. We have found that the boson $s$-paired phase from Fig. 3 and Sec. III C is reproduced roughly when the $d_{2}$ BCS problem is in the weak-pairing regime for both the bonding and antibonding bands and has predominant interchain pairing. Note that the above $d_{1}-d_{2}$ motivation of the $\operatorname{det} \times \mathrm{Pf}$ content is very loose and is hardly inspired by any gauge theory thinking but rather found by trial and error. We also mention that there are likely other ways to construct such paired boson phases. For example, coming from two dimensions, where paired bosons can form a true condensate, one natural construction to explore could be Pf $\times$ Pf. Nevertheless, the det $\times$ Pf that we studied gives us complementary confidence in the DMRG identification of the $s$-wave-paired phase.
${ }^{1}$ P. A. Lee, N. Nagaosa, and X.-G. Wen, Rev. Mod. Phys. 78, 17 (2006).

${ }^{2}$ N. Read and S. Sachdev, Phys. Rev. Lett. 66, 1773 (1991).

${ }^{3}$ X.-G. Wen, Phys. Rev. B 44, 2664 (1991).

${ }^{4}$ T. Senthil and M. P. A. Fisher, Phys. Rev. B 63, 134521 (2001).

${ }^{5}$ R. Moessner and S. L. Sondhi, Phys. Rev. Lett. 86, 1881 (2001).

${ }^{6}$ X.-G. Wen, Phys. Rev. B 65, 165113 (2002).

${ }^{7}$ W. Rantner and X.-G. Wen, Phys. Rev. B 66, 144501 (2002).

${ }^{8}$ M. Hermele, T. Senthil, M. P. A. Fisher, P. A. Lee, N. Nagaosa, and X.-G. Wen, Phys. Rev. B 70, 214437 (2004).

${ }^{9}$ G. Baskaran, Z. Zou, and P. W. Anderson, Solid State Commun. 63, 973 (1987).

${ }^{10}$ L. B. Ioffe and A. I. Larkin, Phys. Rev. B 39, 8988 (1989).

${ }^{11}$ P. A. Lee and N. Nagaosa, Phys. Rev. B 46, 5621 (1992).

${ }^{12}$ J. Polchinski, Nucl. Phys. B 422, 617 (1994).

${ }^{13}$ B. L. Altshuler, L. B. Ioffe, and A. J. Millis, Phys. Rev. B 50, 14048 (1994).

${ }^{14}$ Y. Shimizu, K. Miyagawa, K. Kanoda, M. Maesato, and G.
Saito, Phys. Rev. Lett. 91, 107001 (2003).

${ }^{15}$ O. I. Motrunich, Phys. Rev. B 72, 045105 (2005).

${ }^{16}$ S.-S. Lee and P. A. Lee, Phys. Rev. Lett. 95, 036403 (2005).

${ }^{17}$ O. I. Motrunich and M. P. A. Fisher, Phys. Rev. B 75, 235116 (2007).

${ }^{18}$ T. Senthil, Phys. Rev. B 78, 035103 (2008); 78, 045109 (2008).

${ }^{19}$ D. H. Kim and P. A. Lee, Ann. Phys. (N.Y.) 272, 130 (1999).

${ }^{20}$ Y. Hosotani, J. Phys. A 30, L757 (1997); 31, 7415 (1998).

${ }^{21}$ C. Mudry and E. Fradkin, Phys. Rev. B 50, 11409 (1994).

${ }^{22}$ D. A. Ivanov and P. A. Lee, Phys. Rev. B 57, 2118 (1998).

${ }^{23}$ S. R. White, Phys. Rev. Lett. 69, 2863 (1992); Phys. Rev. B 48, 10345 (1993).

${ }^{24}$ U. Schollwöck, Rev. Mod. Phys. 77, 259 (2005).

${ }^{25}$ D. M. Ceperley, G. V. Chester, and M. H. Kalos, Phys. Rev. B 16, 3081 (1977).

${ }^{26}$ C. S. Hellberg and E. J. Mele, Phys. Rev. Lett. 67, 2080 (1991); 68, 3111 (1992); Phys. Rev. B 48, 646 (1993).

${ }^{27}$ Y. C. Chen and T. K. Lee, Phys. Rev. B 47, 11548 (1993); 54, 
9062 (1996)

${ }^{28}$ H.-Y. Yang and T. Li, J. Phys.: Condens. Matter 19, 186218 (2007).

${ }^{29}$ In this small system with eight bosons (Fig. 7), there is also an abrupt change inside the DBL phase where the ground-state momentum jumps from $\left(k_{x}, k_{y}\right)=(0,0)$ to $\left(k_{x}, k_{y}\right)=(2 \pi / 3, \pi)$ at around $K \approx 2.7 \mathrm{~J}$. We can understand these quantum numbers from specific orbital occupations in the DBL wave-function approach, and the change in the total ground-state momentum arises from a change in orbital occupations in the $d_{2}$ bands. Near $K \approx 2$ VMC finds the optimal DBL state to be $\operatorname{det}_{1}\left[N_{1}^{(0)}\right.$ $\left.=8, N_{1}^{(\pi)}=0\right]_{\mathrm{abc}} \times \operatorname{det}_{2}\left[N_{2}^{(0)}=6, N_{2}^{(\pi)}=2\right]_{\mathrm{abc}}$. The label "abc" stands for antiperiodic boundary conditions when specifying the mean-field orbitals. The total bosonic wave function satisfies periodic boundary conditions and carries momentum $(0,0)$. For the larger $K$, VMC finds the optimal DBL state $\operatorname{det}_{1}\left[N_{1}^{(0)}\right.$ $\left.=8, N_{1}^{(\pi)}=0\right] \times \operatorname{det}_{2}\left[N_{2}^{(0)}=5, N_{2}^{(\pi)}=3\right]$, where now the orbitals are specified with periodic boundary conditions. This state carries momentum $\left(k_{x}, k_{y}\right)=(2 \pi / 3, \pi)$.

${ }^{30}$ Note that $\Delta E$ in Fig. 11 maintains a positive sign throughout the DBL phase, which can be understood roughly as follows: A boson propagates via a bonding mode (with wave vector $q_{x}^{(0)}$ ) in one half and via an antibonding mode $\left(q_{x}^{(\pi)}\right)$ in the other half.
The total phase accumulated by going around the system is $\left(q_{x}^{(0)}+q_{x}^{(\pi)}\right) L_{x} / 2=\pi \rho L_{x}=0 \bmod 2 \pi$ for $\rho=1 / 3$ and the shown $L_{x}$ which are multiples of 6 . Thus, the zero twist boundary condition provides the best (crude) matching for all $K$. When we tried different $L_{x}$ (not shown), the $\Delta E$ could be of either sign.

${ }^{31}$ S. R. White and I. Affleck, Phys. Rev. B 54, 9862 (1996).

${ }^{32}$ A. A. Nersesyan, A. O. Gogolin, and F. H. L. Essler, Phys. Rev. Lett. 81, 910 (1998).

${ }^{33}$ A. D. Klironomos, J. S. Meyer, T. Hikihara, and K. A. Matveev, Phys. Rev. B 76, 075302 (2007).

${ }^{34}$ S. Daul and R. M. Noack, Phys. Rev. B 61, 1646 (2000).

${ }^{35}$ M. Capello, F. Becca, M. Fabrizio, S. Sorella, and E. Tosatti, Phys. Rev. Lett. 94, 026406 (2005).

${ }^{36}$ G. I. Japaridze, R. M. Noack, D. Baeriswyl, and L. Tincani, Phys. Rev. B 76, 115118 (2007).

${ }^{37}$ A. F. Albuquerque et al., J. Magn. Magn. Mater. 310, 1187 (2007).

${ }^{38}$ R. Shankar, Acta Phys. Pol. B 26, 1835 (1995).

${ }^{39}$ H.-H. Lin, L. Balents, and M. P. A. Fisher, Phys. Rev. B 56, 6569 (1997); 58, 1794 (1998).

${ }^{40}$ J. O. Fjaerestad and J. B. Marston, Phys. Rev. B 65, 125106 (2002); J. B. Marston, J. O. Fjaerestad, and A. Sudbo, Phys. Rev. Lett. 89, 056404 (2002). 